\title{
Biotic and abiotic factors influencing soil properties across a latitudinal gradient in Victoria Land, Antarctica
}

\author{
N. Cannone ${ }^{\mathrm{a}, *}$, D. Wagner ${ }^{\mathrm{b}}$, H.W. Hubberten ${ }^{\mathrm{b}}$, M. Guglielmin ${ }^{\mathrm{c}}$ \\ ${ }^{a}$ Department of Biology and Evolution, University of Ferrara, Corso Ercole I d'Este, 32 - 44100 - Ferrara - Italy \\ ${ }^{\mathrm{b}}$ Alfred Wegener Institute for Polar and Marine Research, Potsdam, Germany \\ ${ }^{c} D B S F$, University Insubria, Varese, Italy
}

\begin{abstract}
Through the cooperative efforts of the Scientific Committee on Antarctic Research (SCAR) Evolution and Biodiversity in Antarctica (EBA) Project and the Latitudinal Gradient Project (LGP), a monitoring network was established in Victoria Land in 2002 to assess the impacts of climate change on vegetation, soils, active-layer dynamics, and permafrost across a latitudinal gradient. In this study, we report on the key factors influencing soil development across the gradient, including vegetation, parent material characteristics, and climate. Physical and chemical soil properties at depths of 2-8 and 10-20 cm were investigated at 7 sites and on 14 permanent plots from Apostrophe Island in Northern Victoria Land $\left(73^{\circ} 30^{\prime} \mathrm{S}, 167^{\circ} 50^{\prime} \mathrm{E}\right)$ to Granite Harbour in Southern Victoria Land $\left(77^{\circ} 00^{\prime} \mathrm{S}, 162^{\circ} 26^{\prime} \mathrm{E}\right)$ along the Ross Sea coast. The relationships among vegetation, parent material, and regional climate and soil properties were tested with Principal Component Analyses. There were no significant correlations or relationships in soil properties across the climate gradient. In fact, local microclimatic appears to be more effective than the regional gradient in influencing the properties. Microclimate was also important relative to active-layer depth and vegetation distribution. Lithology was strongly related to several chemical parameters, notably extractable $\mathrm{Al}, \mathrm{Fe}, \mathrm{Ca}, \mathrm{K}$, but was unrelated to grain-size distribution. Vegetation was related to the chemistry of the surface-soil layer, including nitrate, organic carbon, $\mathrm{C} / \mathrm{N}$ ratio and water content, and also the active-layer depth. Penguins had the greatest influence on soil properties in initiating the development of ornithogenic soils. Further analyses on soil properties, including a greater number of sites, will be required to represent more extensively the lithological variability and to extend the latitudinal extremes of the gradient. The results presented here are an important reference for future monitoring activities in Victoria Land.
\end{abstract}

(C) 2007 Elsevier B.V. All rights reserved.

Keywords: Soil; Vegetation; Active layer; Parent material; Monitoring network; Climate change; Antarctica

\section{Introduction}

Polar regions are important for assessing and monitoring climate change impacts on biotic and abiotic components of Antarctic ecosystems (i.e. Convey, 2001; Doran et al., 2002; Geringhausen et al., 2003; Vaughan et al., 2003; Cook et al., 2005; Turner et al., 2005). Changes in climate may affect vegetation, soil properties, active-layer dynamics, and permafrost in different ways and these environmental components may interact through complex mechanisms, activating both positive and negative feedbacks. These topics have been studied only recently in Antarctica (Melick and Seppelt, 1997; Barrett et

\footnotetext{
* Corresponding author. Tel.: +39 0532 293785; fax: +39 0532208561.

E-mail address: nicoletta.cannone@unife.it (N. Cannone).
}

al., 2006a, 2006b; Beyer et al., 2000; Cannone et al., 2006; Guglielmin et al., 2005, 2008-this issue; Bockheim, 2008-this issue). Moreover, vegetation and active layer dynamics play an important role in the carbon cycle (cf., Oechel et al., 2000; Mack et al., 2004; Chapin et al., 2005), although the carbon content of Antarctic soils is much lower than in the Arctic.

In Antarctica, international panels are addressing the problem of how to detect the effects of climate change on ecosystems. In particular, within the framework of the Scientific Committee on Antarctic Research (SCAR) Evolution and Biodiversity in Antarctica (EBA) program, formerly the Regional Sensitivity to Climate Change in Antarctic terrestrial and limnetic ecosystems (RiSCC) project, specific research protocols have been developed for assessing and monitoring climate change impacts on vegetation, soils, and permafrost. Their main approach is to set 
up networks along latitudinal and environmental gradients (Cannone and Guglielmin, 2003; Cannone, 2004, 2006).

In the frame of the SCAR project EBA and, in cooperation with the Latitudinal Gradient Project (LGP), a monitoring network was established in 2002 and 2003 to assess the impacts of climate change on vegetation, soils, and permafrost in Victoria Land (Continental Antarctica) across a latitudinal gradient (Cannone, 2006). The network includes 7 sites and 14 permanent plots and is located along a latitudinal gradient from Apostrophe Island $\left(73^{\circ} 30^{\prime} \mathrm{S}, 167^{\circ} 50^{\prime} \mathrm{E}\right)$ in Northern Victoria Land to Finger Point (Granite Harbour) $\left(77^{\circ} 00^{\prime} \mathrm{S}, 162^{\circ} 26^{\prime} \mathrm{E}\right)$ in Southern Victoria Land. Plots are located at key sites along this gradient so as to include a coastal transect and a coast-inland sub-transect in the Terra Nova Bay area. The study sites were selected on the basis of the results of a latitudinal-transect study that investigated 25 sites from Cape Hallett $\left(72^{\circ} 76^{\prime} \mathrm{S}\right.$, $\left.169^{\circ} 56^{\prime} \mathrm{E}\right)$ to Lake Fryxell $\left(77^{\circ} 35^{\prime} \mathrm{S}, 163^{\circ} 20^{\prime} \mathrm{E}\right)$ (Cannone, 2005).

Here we report on work carried out at seven coastal transect sites in Victoria Land to analyze changes in physical and chemical properties of soils in relation to parent material and vegetation conditions along the climatic gradient. The main aims of the paper are to:

1) describe the main physical and chemical parameters of the soils within the monitoring network as a reference for future monitoring activities;
2) assess the relationships between the soil characteristics and a) the latitudinal gradient, b) the lithology, c) the vegetation.

\section{Study Sites}

The study area is located in Victoria Land, East Antarctica where, within the main latitudinal network, seven sites and 14 permanent plots were investigated from Apostrophe Island $\left(73^{\circ} 30^{\prime} \mathrm{S}, 167^{\circ} 50^{\prime} \mathrm{E}\right)$ to Finger Point (Granite Harbour) $\left(77^{\circ} 00^{\prime} \mathrm{S}\right.$, $162^{\circ} 26^{\prime} \mathrm{E}$ ) (Fig. 1). The climate of Victoria Land is a frigid Antarctic one, with a gradient of increasing mean annual air temperature (MAAT) from (a) Southern Victoria Land, with MAAT of $-19.9^{\circ} \mathrm{C}$ at Scott Base $\left(77^{\circ} 51^{\prime} \mathrm{S} ; 167^{\circ} 46^{\prime} \mathrm{E}\right)$ over the period 1957-2005 (Harper, unpublished), to (b) Boulderclay $\left(74^{\circ} 43^{\prime} \mathrm{S}, 164^{\circ} 05^{\prime} \mathrm{E}\right)$, in the vicinity of Mario Zucchelli Station (Terra Nova Bay area), with MAT of $-16.4{ }^{\circ} \mathrm{C}$ to $-15.1{ }^{\circ} \mathrm{C}$ in the period 1997-2003 (Guglielmin, 2006), to (c) Cape Hallett, where the MAT is c. $-15.3^{\circ} \mathrm{C}$ (Duphorn, 1981). Precipitation, always in the form of snow, is very low and ranges between 100 and $200 \mathrm{~mm}$ (Grigioni et al., 1992).

All sites are located along the coast, with the exception of Tarn Flat, which is inland on the slope area before the plateau. They have a similar altitudinal range $(10-250 \mathrm{~m}$ a.s.l. $)$ so that comparable data, not influenced by elevation, are obtained for assessing relationships along the latitudinal gradient. The sites represent the most common environmental conditions, and almost all the rock substrates were sampled (i.e. granite, basalts,

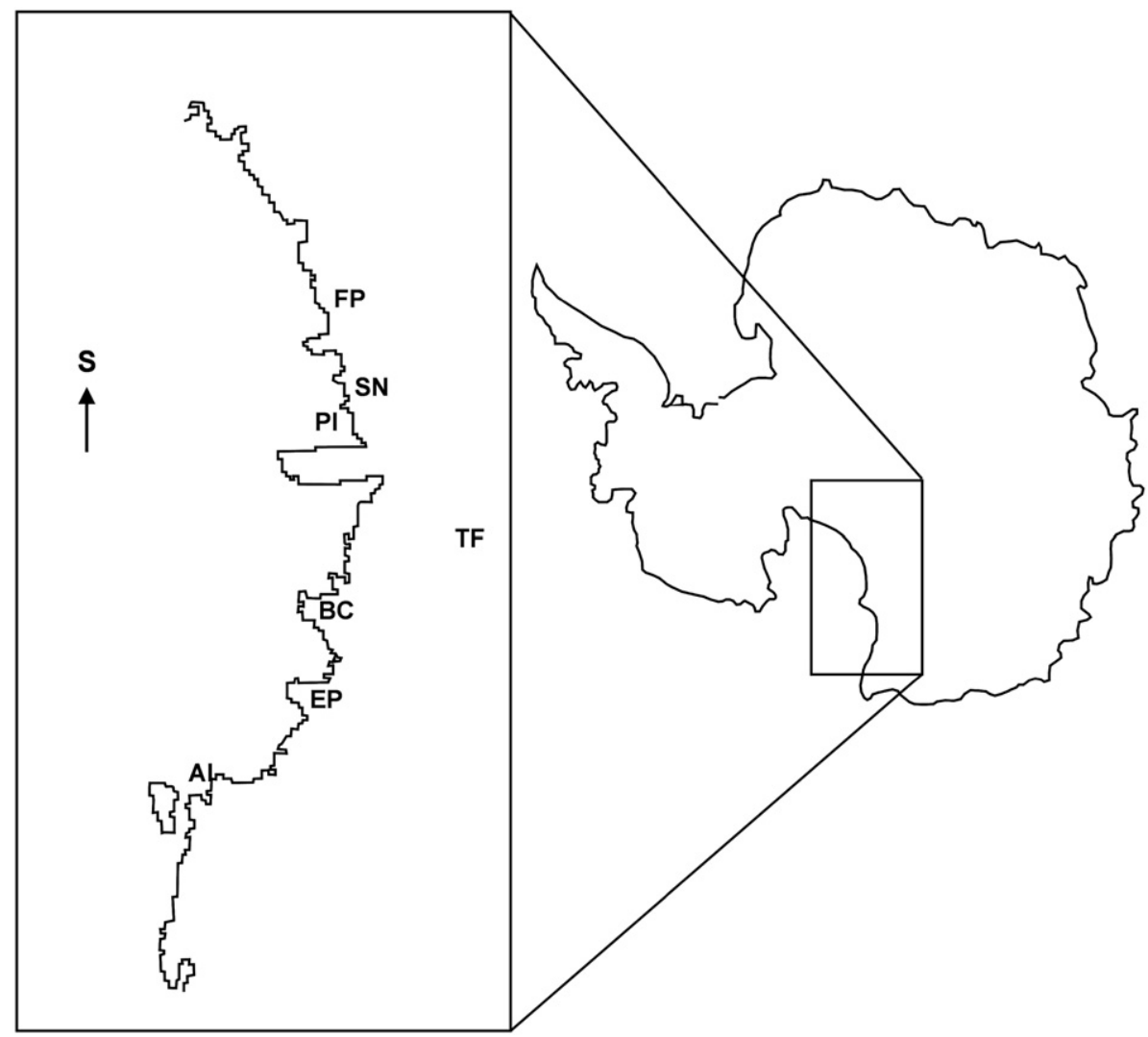

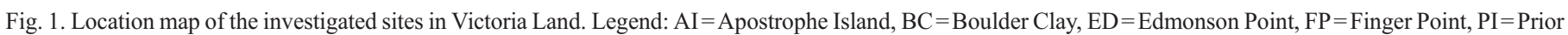
Island, $\mathrm{SN}=$ Starr Nunatak, $\mathrm{TF}=$ Tarn Flat. 
gabbro, metamorphic rocks, morainic and marine deposits). Some sites have ornithogenic soils. All the sites are located in ice-free areas, most of them very close to glacial boundaries, and are characterized by the occurrence of continuous permafrost with an active layer thickness ranging from $20 \mathrm{~cm}$ to more than $100 \mathrm{~cm}$ (Guglielmin and French, 2004), and, in one case (Apostrophe Island, permanent plot 7), by the occurrence of patterned ground.

The vegetation of Victoria Land is composed exclusively of cryptogams. Previous descriptions of the moss and lichen flora and of the main vegetation communities have been provided (Kappen, 1985; Wynn-Williams, 1985; Schwarz et al., 1992; Castello and Nimis, 1995; Seppelt et al., 1995, 1996; Seppelt and Green, 1998; Smith, 1999; Cannone, 2005). The vegetation of the Victoria Land monitoring network includes four main vegetation types, respectively dominated by a) mosses, b) mosses encrusted by epiphytic lichens, c) macrolichens, and d) scattered epilithic lichens and mosses. Cannone (2006) described the vegetation of the permanent plots of the monitoring network in detail.

The soils of Victoria Land are Gelisols: while in the Dry Valleys of Southern Victoria Land Anhyortels and Anhyturbels are prevalent (Bockheim, 2002), in Northern Victoria Land data on the soil classification are still lacking.

\section{Methods}

\subsection{Field}

At each permanent plot, pits were dug for describing and sampling soils. Due to the thin active layer, the very coarse texture, and logistical problems, the maximum depth of the pits was $55 \mathrm{~cm}$. To obtain comparable data from all the investigated sites and plots, soils were sampled at two depths, from 2 to $8 \mathrm{~cm}$ and from 10 to $20 \mathrm{~cm}$. Where there was evidence of accumulation of organic matter at the surface, a surface layer (between 1 and $3 \mathrm{~cm}$ ) was collected. Soil samples were immediately stored at $-20{ }^{\circ} \mathrm{C}$ and sent in frozen state to the Alfred Wegener Institute (AWI) laboratory for the chemical and physical analyses.

Maximum thawing depth at the moment of the sampling (from mid December to mid January) was estimated by using a frost probe according the CALM (Circumpolar Active Layer Monitoring) protocol (Nelson et al., 1998) at least on 5 different points within the plot and excluding all the measurements hindered by the occurrence of stones in the ground. In some sites also the temperature was measured, according to Guglielmin (2006), to estimate the maximum depth of the permafrost table $\left(0{ }^{\circ} \mathrm{C}\right.$ isotherm $)$.

\subsection{Laboratory}

Soil samples were oven dried at $105{ }^{\circ} \mathrm{C}$ for 24 hours. The water content was calculated by taking the difference between the fresh sample weight and that of the dried sample. Particlesize analysis was performed by sieving for gravel and sand, and using a hydrometer for silt and clay (Day, 1965). All chemical analyses were carried out on the $<2 \mathrm{~mm}$ soil fraction. Watersoluble cations and anions were extracted by a modified method of the Gerasimov and Glazovskaya (1965). A 1:1 soil to distilled water suspension was used for determining $\mathrm{pH}$. Electric conductivity was carried out using a conductivity bridge and cell. Total carbon (TC) and total nitrogen (TN) contents were determined with an automatic element analyser (Elementar VARIO EL III). The total organic carbon (TOC) content was measured on corresponding samples after $\mathrm{HCl}(10 \%)$ acid digestion to remove the carbonate on the same analyser (Elementar VARIO EL III). The $\mathrm{C} / \mathrm{N}$ ratio was calculated on the basis of TOC and TN values. The dried original sample $(2 \mathrm{~g})$ was diluted with $50 \mathrm{ml}$ of distilled water and, after 3 minutes stirring and filtration through a $0.45-\mu \mathrm{m}$ cellulose acetate filter. Water-extractable cations were analysed with an Inductively Coupled Plasma Optical Emission Spectrometer (ICP-OES, Perkins Elmer) and anions with an Ion Chromatograph (DX320, DIONEX Corp., USA). For all chemical analyses analytical precision was checked using international standards (NBS, SRM).

The number of chemo-organotrophic bacteria was determined on agar plates using a 10 -fold serial dilution of sediment in growth medium. The agar medium was prepared according to the protocol of Bunt and Rovina (1955) for Antarctic soils. For each sample, three agar plates were inoculated with $0.1 \mathrm{ml}$ from each dilution and incubated for 3 weeks at $18{ }^{\circ} \mathrm{C}$. Cell numbers were determined by counting the colonies and taking into account the individual dilution steps.

\subsection{Statistical analyses}

Pearson correlation matrices were calculated by means of the software Statistica 6.0 made by StatSoft ${ }^{\circledR}$, and Principal Component Analyses (PCA) were performed using the software CANOCO for Windows (ter Braak and Šmilauer, 1998). In particular, for all PCA the data were log transformed, the scaling was made through the inter-species correlation, the standardization by species was centered, and the sample standardization was centered and normal. A table with the eigenvalues and the percentage of variance was reported for each analysis.

\section{Results}

Soil properties were analyzed in relation to different abiotic and biotic environmental factors, including site location, latitudinal (and associated climatic) gradient, lithology, and vegetation.

\subsection{Soil properties in relation to site}

Seven sites, one inland and six coastal, were investigated (Table 1). No evidence of cryoturbation was observed in the investigated sites, and only in correspondence with AI7 did a frost-fissure polygon occur. Holocene raised beaches were not included in the selected sites. Generally the sites are far both from streams and ponds or lakes, except for Edmonson Point. Ornithogenic soils occur at the PP6 site at Prior Island, although 
Table 1

Location and main characteristics of the study sites * active layer thickness obtained by thermal data

\begin{tabular}{|c|c|c|c|c|c|c|c|c|c|c|c|c|}
\hline Sites & Coordinates & $\begin{array}{l}\text { Elevation } \\
\text { (m a.s.1.) }\end{array}$ & $\begin{array}{l}\text { Plot } \\
\text { Number }\end{array}$ & Location & Lithology & $\begin{array}{l}\text { Slope } \\
\text { and } \\
\text { Aspect }\end{array}$ & $\begin{array}{l}\text { Active } \\
\text { Layer } \\
(\mathrm{cm})\end{array}$ & $\begin{array}{l}\text { Vegetation } \\
\text { Coverage } \\
(\%)\end{array}$ & Vegetation Type & Soil sample label & $\begin{array}{l}\text { Soil sample } \\
\text { depth }(\mathrm{cm})\end{array}$ & $\begin{array}{l}\text { Soil } \\
\text { classification } \\
\text { (ST) }\end{array}$ \\
\hline Apostrophe Island & $73^{\circ} 30^{\prime} \mathrm{S} 167^{\circ} 50^{\prime} \mathrm{E}$ & 50 & PP7 & Coast & $\begin{array}{l}\text { Morainic deposits } \\
\text { (Gabbro) }\end{array}$ & $2^{\circ} ; \mathrm{S}$ & 43 & 53.4 & $\begin{array}{l}\text { Macrolichens } \\
\text { (Usnea sphacelata) }\end{array}$ & A17_1 A17_2 & $3-8 \quad 12-18$ & $\begin{array}{l}\text { Glacic } \\
\text { Haploturbels }\end{array}$ \\
\hline Apostrophe Island & $73^{\circ} 30^{\prime} \mathrm{S} 167^{\circ} 50^{\prime} \mathrm{E}$ & 50 & PP8 & Coast & $\begin{array}{l}\text { Morainic deposits } \\
\text { (Gabbro) }\end{array}$ & $3^{\circ} ; \mathrm{W}$ & 45 & 4.8 & $\begin{array}{l}\text { Scattered epilithic } \\
\text { lichens and mosses } \\
\text { (Bryum argenteum) }\end{array}$ & A18_1 A18_2 & $0-66-21$ & $\begin{array}{l}\text { Glacic } \\
\text { Haplorthels }\end{array}$ \\
\hline Edmonson Point & $74^{\circ} 19^{\prime} \mathrm{S} 165^{\circ} 07^{\prime} \mathrm{E}$ & 40 & PP1 & Coast & $\begin{array}{l}\text { Alluvial sediments } \\
\text { (Basalts) }\end{array}$ & $2^{\circ} ; \mathrm{E}$ & $41^{*}$ & 83.8 & $\begin{array}{l}\text { Mosses (Bryum } \\
\text { argenteum, } \\
\text { B. pseudotriquetrum, } \\
\text { Ceratodon purpureus) }\end{array}$ & EP1_1 EP1_1b & $3-53-5$ & $\begin{array}{l}\text { Aquic } \\
\text { Psammorthels }\end{array}$ \\
\hline Edmonson Point & $74^{\circ} 19^{\prime} \mathrm{S} 165^{\circ} 07^{\prime} \mathrm{E}$ & 40 & PP2 & Coast & $\begin{array}{l}\text { Alluvial sediments } \\
\text { (Basalts) }\end{array}$ & $3^{\circ} ; \mathrm{E}$ & $82 *$ & 88.1 & $\begin{array}{l}\text { Mosses (Syntrichia } \\
\text { princeps) }\end{array}$ & EP2_1 EP2_2 & $3-7 \quad 12-16$ & $\begin{array}{l}\text { Aquic } \\
\text { Haplorthels }\end{array}$ \\
\hline Edmonson Point & $74^{\circ} 19^{\prime} \mathrm{S} 165^{\circ} 07^{\prime} \mathrm{E}$ & 50 & PP3 & Coast & $\begin{array}{l}\text { Weathering bedrock } \\
\text { (Basalts) }\end{array}$ & $2^{\circ} ; \mathrm{E}$ & $48^{*}$ & 0 & No vegetation & EP3_1 EP3_2 & $3-612-15$ & $\begin{array}{l}\text { Lithic } \\
\text { Haplorthels }\end{array}$ \\
\hline Boulder Clay & $74^{\circ} 30^{\prime} \mathrm{S} 164^{\circ} 05^{\prime} \mathrm{E}$ & 150 & PP10 & Coast & $\begin{array}{l}\text { Morainic deposits } \\
\text { (granite) }\end{array}$ & $2^{\circ} ; \mathrm{E}$ & 62 & 11.2 & $\begin{array}{l}\text { Scattered mosses } \\
\text { (Bryum subrotundifolium, } \\
\text { Schistidium antarctici) } \\
\text { and epilithic lichens }\end{array}$ & BC10_1 BC10 $\_2$ & $1-33-9$ & $\begin{array}{l}\text { Glacic } \\
\text { Haplorthels }\end{array}$ \\
\hline Tarn Flat & $74^{\circ} 59^{\prime} \mathrm{S} 162^{\circ} 37^{\prime} \mathrm{E}$ & 100 & PP14 & Inland & $\begin{array}{l}\text { Morainic deposits } \\
\text { (granite) }\end{array}$ & $5^{\circ} ; \mathrm{S}$ & 42 & 0.8 & $\begin{array}{l}\text { Scattered epilithic } \\
\text { crustose lichens }\end{array}$ & TF14_1 TF14_2 & $2-410-15$ & $\begin{array}{l}\text { Glacic } \\
\text { Haplorthels }\end{array}$ \\
\hline Tarn Flat & $74^{\circ} 59^{\prime} \mathrm{S} 162^{\circ} 37^{\prime} \mathrm{E}$ & 20 & PP17 & Inland & $\begin{array}{l}\text { Morainic deposits } \\
\text { (granite) }\end{array}$ & $2^{\circ} ; \mathrm{W}$ & $58^{*}$ & 1.3 & $\begin{array}{l}\text { Scattered epilithic } \\
\text { crustose lichens }\end{array}$ & TF17_1 TF17_2 & $1-3 \quad 10-15$ & $\begin{array}{l}\text { Glacic } \\
\text { Psammorthels }\end{array}$ \\
\hline Prior Island & $75^{\circ} 41^{\prime} \mathrm{S} 162^{\circ} 52^{\prime} \mathrm{E}$ & 150 & PP4 & Coast & $\begin{array}{l}\text { Morainic deposits } \\
\text { (granite) }\end{array}$ & $2^{\circ} ; \mathrm{E}$ & 55 & 46.5 & $\begin{array}{l}\text { Mosses (Schistidium } \\
\text { antarctici, Sarconeurum } \\
\text { glaciale) encrusted by lichens } \\
\text { (Candelariella, Xanthoria) }\end{array}$ & PP4_0 & $0-3$ & $\begin{array}{l}\text { Glacic } \\
\text { Haplorthels }\end{array}$ \\
\hline Prior Island & $75^{\circ} 41^{\prime} \mathrm{S} 162^{\circ} 52^{\prime} \mathrm{E}$ & 150 & PP6 & Coast & $\begin{array}{l}\text { Morainic deposits } \\
\text { (granite) }\end{array}$ & $3^{\circ} ; \mathrm{SE}$ & 47 & 10.9 & $\begin{array}{l}\text { Scattered epilithic lichens } \\
\text { (Buellia frigida, Xanthoria) }\end{array}$ & $\begin{array}{l}\text { PP6_0 PP6_1 PP6_2 } \\
\text { PP6_3 PP6_3b }\end{array}$ & 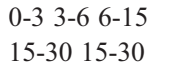 & $\begin{array}{l}\text { Glacic } \\
\text { Haplorthels }\end{array}$ \\
\hline Starr Nunatak & $75^{\circ} 53^{\prime} \mathrm{S} 162^{\circ} 33^{\prime} \mathrm{E}$ & 150 & PP15 & Coast & $\begin{array}{l}\text { Morainic deposits } \\
\text { (granite) }\end{array}$ & $2^{\circ} ; \mathrm{NE}$ & 65 & 60.8 & $\begin{array}{l}\text { Mosses (Sarconeurum } \\
\text { glaciale) encrusted by } \\
\text { lichens (Physcia caesia) }\end{array}$ & ST15_1 ST15_2 & $2-710-16$ & $\begin{array}{l}\text { Glacic } \\
\text { Haplorthels }\end{array}$ \\
\hline Starr Nunatak & $75^{\circ} 53^{\prime} \mathrm{S} 162^{\circ} 33^{\prime} \mathrm{E}$ & 150 & PP16 & Coast & $\begin{array}{l}\text { Morainic deposits } \\
\text { (granite) }\end{array}$ & $2^{\circ} ; \mathrm{SW}$ & $82 *$ & 2.8 & $\begin{array}{l}\text { Scattered epilithic lichens } \\
\text { (Buellia frigida, } \\
\text { Lecidella siplei) }\end{array}$ & $\begin{array}{l}\text { ST16_1 ST16_2 } \\
\text { ST16_3 }\end{array}$ & $2-4 \quad 7-1322-27$ & $\begin{array}{l}\text { Glacic } \\
\text { Haplorthels }\end{array}$ \\
\hline Finger Point & $77^{\circ} 00^{\prime} \mathrm{S} 162^{\circ} 26^{\prime} \mathrm{E}$ & $20-50$ & PP9 & Coast & $\begin{array}{l}\text { Morainic deposits } \\
\text { (granite) }\end{array}$ & $3^{\circ} ; \mathrm{E}$ & 62 & 95.8 & $\begin{array}{l}\text { Mosses (Schistidium } \\
\text { antarctici, Bryum spp.) } \\
\text { encrusted by lichens } \\
\text { (Lecidella siplei) }\end{array}$ & FP9_0 FP9_1 FP9_2 & $2-47-11 \quad 20-25$ & $\begin{array}{l}\text { Glacic } \\
\text { Haplorthels }\end{array}$ \\
\hline Finger Point & $77^{\circ} 00^{\prime} \mathrm{S} 162^{\circ} 26^{\prime} \mathrm{E}$ & $20-50$ & PP19 & Coast & $\begin{array}{l}\text { Morainic deposits } \\
\text { (granite) }\end{array}$ & $2^{\circ} ; \mathrm{N}$ & 73 & 0.7 & $\begin{array}{l}\text { Scattered epilithic } \\
\text { lichens (Lecidella siplei) }\end{array}$ & $\begin{array}{l}\text { FP19_0 FP19_1 } \\
\text { FP19_2 }\end{array}$ & $0-4 \quad 5-9 \quad 14-21$ & $\begin{array}{l}\text { Glacic } \\
\text { Psammorthels }\end{array}$ \\
\hline
\end{tabular}


Table 2

Chemical and physical soil parameters of the investigated sites. Bacteria are calculated as cell number per gram (number/g) divided by 100

\begin{tabular}{|c|c|c|c|c|c|c|c|c|c|c|c|c|c|c|c|c|c|c|c|c|c|c|c|c|c|}
\hline Sample & $\mathrm{pH}$ & $\begin{array}{l}\text { Cond } \\
(\mu \mathrm{s} / \mathrm{cm})\end{array}$ & $\mathrm{F}^{-}(\mathrm{mg} / \mathrm{l})$ & $\mathrm{Cl}^{-}(\mathrm{mg} / \mathrm{l})$ & $\begin{array}{l}\mathrm{SO}_{4}^{-} \\
(\mathrm{mg} / \mathrm{l})\end{array}$ & $\begin{array}{l}\mathrm{Br}^{-} \\
(\mathrm{mg} / \mathrm{l})\end{array}$ & $\begin{array}{l}\mathrm{NO}_{3}^{-} \\
(\mathrm{mg} / \mathrm{l})\end{array}$ & $\begin{array}{l}\mathrm{PO}_{4}^{-} \\
(\mathrm{mg} / \mathrm{l})\end{array}$ & $\begin{array}{l}\mathrm{HCO}_{3}^{-} \\
(\mathrm{mg} / \mathrm{l}) \\
\end{array}$ & $\begin{array}{l}\mathrm{Al} \\
(\mu \mathrm{g} / \mathrm{l})\end{array}$ & $\begin{array}{l}\mathrm{Ca} \\
(\mathrm{mg} / \mathrm{l})\end{array}$ & $\begin{array}{l}\mathrm{Fe} \\
(\mu \mathrm{g} / \mathrm{l})\end{array}$ & $\begin{array}{l}\mathrm{K} \\
(\mathrm{mg} / \mathrm{l})\end{array}$ & $\begin{array}{l}\mathrm{Mg} \\
(\mathrm{mg} / \mathrm{l})\end{array}$ & $\begin{array}{l}\mathrm{Na} \\
(\mathrm{mg} / \mathrm{l})\end{array}$ & $\begin{array}{l}\mathrm{P} \\
(\mathrm{mg} / \mathrm{l})\end{array}$ & $\begin{array}{l}\mathrm{Si} \\
(\mathrm{mg} / \mathrm{l})\end{array}$ & $\begin{array}{l}\text { BacteriaCell } \\
\text { (n) }\end{array}$ & $\begin{array}{l}\text { Corg } \\
(\%)\end{array}$ & $\begin{array}{l}\mathrm{N} \\
(\%)\end{array}$ & $\mathrm{C} / \mathrm{N}$ & $\begin{array}{l}\text { Sand } \\
(\%)\end{array}$ & $\begin{array}{l}\text { Silt } \\
(\%)\end{array}$ & $\begin{array}{l}\text { Clay } \\
(\%)\end{array}$ & $\begin{array}{l}\text { Water } \\
(\%)\end{array}$ \\
\hline AI7_1 & 5.5 & 15.2 & 0.3 & 1.8 & 1 & $<0.1$ & 1.1 & 0.4 & 2.3 & 89.9 & $<0.1$ & 33.7 & 0.3 & $<0.1$ & 2.7 & 0.1 & 0.5 & 798 & 0.3 & 0.1 & 3.2 & 75,7 & 22,5 & 1,8 & 7 \\
\hline AI7_2 & 5.6 & 12 & 0.2 & 1.2 & 0.6 & $<0.1$ & 0.9 & 0.5 & 2.9 & 35.3 & $<0.1$ & $<20$ & 0.4 & $<0.1$ & 2.1 & 0.1 & 0.8 & 953 & 0.3 & 0.1 & 4 & 80,9 & 17,3 & 1,8 & 7.9 \\
\hline AI8_1 & 5.5 & 14.4 & 0.1 & 1.2 & 0.7 & $<0.1$ & 2.1 & 0.3 & 1.7 & 33.8 & 0.1 & 30.1 & 0.3 & $<0.1$ & 2.1 & 0.1 & 0.5 & 234 & 0.2 & 0.8 & 0.3 & 67,5 & 31,1 & 1,4 & 4.4 \\
\hline AI8_2 & 5.8 & 8 & 0.1 & 0.7 & 0.5 & $<0.1$ & 0.4 & 0.5 & 1.8 & 37.5 & 0.2 & 23.1 & 0.2 & $<0.1$ & 1.3 & 0.1 & 0.7 & 122 & 0.2 & 0.7 & 0.3 & 69,4 & 29,2 & 1,4 & 4 \\
\hline EP1_1 & 5.7 & 25.5 & 0.2 & 1 & 0.9 & $<0.1$ & 7.5 & 0.2 & 3.1 & 30.3 & 0.6 & 24.5 & 0.9 & 0.3 & 3.8 & 0.2 & 0.1 & 17 & 1.6 & 0.2 & 7.4 & 91,5 & 7,5 & 0,9 & 30.3 \\
\hline EP1_1b & 5.5 & 26.2 & 0.2 & 1.5 & 0.9 & $<0.1$ & 7.1 & 0.3 & 2.6 & $<20$ & 0.6 & $<20$ & 1 & 0.3 & 3.7 & 0.1 & $<0.1$ & 59 & & & & & & & \\
\hline EP2_1 & 5.9 & 8.5 & 0.1 & 0.5 & 0.2 & $<0.1$ & 1.5 & 0.1 & 3.2 & 38.7 & $<0.1$ & 29.2 & 0.3 & $<0.1$ & 1.6 & $<0.1$ & 0.2 & 10 & 0.5 & 0.1 & 4.5 & 67,3 & 32,5 & 0,2 & 27.6 \\
\hline EP2_2 & 5.9 & 7 & 0.2 & 0.7 & 0.2 & $<0.1$ & 0.6 & 0.2 & 1.6 & 73.8 & $<0.1$ & 35.1 & 0.2 & $<0.1$ & 1.3 & $<0.1$ & 0.2 & 1553 & 0.2 & 0.6 & 0.3 & 80,5 & 17,6 & 1,9 & \\
\hline EP3_1 & 5 & 25.2 & 0.5 & 4.5 & 1.36 & $<0.1$ & 1.4 & 0.7 & 1 & 42.2 & 0.2 & 72.3 & 0.5 & $<0.1$ & 4.9 & 0.2 & 0.1 & 5546 & 0.1 & 0.1 & 1.9 & 95,8 & 4,2 & 0,0 & 16 \\
\hline EP3_2 & 6 & 17.3 & 0.2 & 3.5 & 0.8 & $<0.1$ & 0.6 & 0.2 & 1.6 & $<20$ & $<0.1$ & $<20$ & 0.3 & $<0.1$ & 3.2 & $<0.1$ & $<0.1$ & 89 & 0.2 & 0.1 & 3.1 & 89,7 & 9,6 & 0,7 & 17.5 \\
\hline BC10_1 & 6 & 14.9 & 0.1 & 1 & 0.4 & $<0.1$ & 2.9 & 0.2 & 3.2 & 134.7 & $<0.1$ & 122.1 & 0.2 & $<0.1$ & 3 & 0.2 & 0.3 & 772 & 0.2 & 0.6 & 0.3 & 89,0 & 7,9 & 3,1 & 0.9 \\
\hline BC10_2 & 6.2 & 7.5 & 0.1 & 0.4 & 0.3 & $<0.1$ & 0.4 & 0.3 & 2.7 & 246.6 & 0.1 & 219 & $<0.2$ & $<0.1$ & 1.7 & $<0.1$ & 0.5 & 585 & 0.2 & 0.6 & 0.3 & 62,5 & 21,4 & 16,2 & 5.1 \\
\hline TF14_1 & 5.5 & 6.9 & 0.1 & 0.3 & 0.6 & $<0.1$ & 1.1 & $<0.1$ & 0.7 & 44.7 & $<0.1$ & 51.2 & $<0.2$ & $<0.1$ & 1.2 & $<0.1$ & 0.1 & 613 & 0.1 & 0.1 & 2 & 95,6 & 4,2 & 0,2 & 5.9 \\
\hline TF14_2 & 5.7 & 4.9 & 0.1 & 0.3 & 0.3 & $<0.1$ & $<0.2$ & $<0.1$ & 1.7 & 28.5 & $<0.1$ & 25.4 & $<0.2$ & $<0.1$ & 0.9 & $<0.1$ & 0.2 & 34 & 0.1 & 0.1 & 1.7 & 84,1 & 14,2 & 1,7 & 5.3 \\
\hline TF17_1 & 5.7 & 20.3 & 0.5 & 1.9 & 0.8 & $<0.1$ & 0.3 & $<0.1$ & 7.3 & 496 & 0.3 & 486.9 & 0.6 & 0.2 & 4.4 & $<0.1$ & 1.4 & 44 & 0.2 & 0.1 & 2.8 & 67,9 & 13,8 & 18,4 & 5.4 \\
\hline TF17_2 & 5.7 & 16.7 & 0.5 & 0.4 & 0.4 & $<0.1$ & $<0.2$ & 0.2 & 8.2 & 401 & 0.5 & 325.6 & 1 & 0.2 & 2.8 & $<0.1$ & 2.1 & 4520 & 0.2 & 0.1 & 2.6 & 67,8 & 11,6 & 20,6 & 2.6 \\
\hline PP4_o & 5 & 70.1 & 0.1 & 3 & 2.4 & $<0.1$ & 24.6 & 2.3 & 1.3 & 29.1 & 1.6 & 25.9 & 4 & 1.1 & 4.3 & 1.1 & $<0.1$ & 215 & 1.2 & 0.2 & 5.5 & 96,1 & 2,1 & 1,8 & \\
\hline PP6_0 & 6.4 & 418 & 1.5 & 100.4 & 6.4 & 0.2 & $<0.2$ & 51 & 38.4 & 69 & 1.2 & 81.1 & 11.2 & 7.2 & 60.2 & 17.8 & 0.2 & 562 & 3.2 & 0.6 & 4.9 & 85,8 & 8,7 & 5,5 & 3.8 \\
\hline PP6_1 & 7.6 & 649 & 6.2 & 62.8 & 8.4 & 0.2 & 22.5 & 285 & 154 & 45.5 & 0.7 & 49.9 & 11.3 & 33.2 & 66.8 & 87.7 & 0.3 & 3206 & 4 & 1.4 & 2.9 & 74,8 & 22,7 & 2,5 & 0.7 \\
\hline PP6_2 & 7.4 & 530 & 4.1 & 46.9 & 9.4 & 0.1 & 11.4 & 207 & 122.6 & 33.4 & 0.4 & 53.9 & 8.6 & 18.7 & 60.5 & 66.5 & 0.2 & 1 & 3.1 & 1.2 & 2.7 & 85,8 & 1,0 & 13,1 & 0.7 \\
\hline PP6_3 & 7.6 & 559 & 3.1 & 42.2 & 22.3 & 0.1 & 19.3 & 206 & 113.6 & 155.5 & 0.6 & 244.8 & 14.7 & 2.32 & 92.6 & 73.3 & 0.4 & 1046 & 0.7 & 0.4 & 1.7 & 88,8 & 7,3 & 3,9 & 1.4 \\
\hline PP6_3b & 7.5 & 448 & 2.3 & 35.9 & 18.6 & 0.1 & 16.4 & 156 & 87.5 & 113 & 0.4 & 172.3 & 11.3 & 1.4 & 76.4 & 52 & 0.3 & 41 & & & & & & & \\
\hline ST15_1 & 5.2 & 9.2 & 0.05 & 0.5 & 0.1 & $<0.1$ & 1.9 & 1.5 & 1.5 & 22.1 & 0.2 & $<20$ & 0.4 & $<0.1$ & 1.1 & 0.5 & $<0.1$ & 836 & 0.9 & 0.2 & 4.6 & 95,8 & 3,0 & 1,2 & 2.9 \\
\hline ST15_2 & 5.5 & 3.9 & $<0.1$ & 0.2 & 0.1 & $<0.1$ & $<0.2$ & 1.1 & 2.2 & $<20$ & $<0.1$ & $<20$ & $<0.2$ & $<0.1$ & 0.5 & 0.3 & $<0.1$ & 109 & 0.2 & 0.1 & 2.9 & 94,9 & 4,6 & 0,5 & 1.5 \\
\hline ST16_1 & 5.7 & 13.6 & $<0.1$ & 1.38 & 0.9 & $<0.1$ & 0.6 & 2.7 & 3.2 & 26.1 & $<0.1$ & 30.3 & 0.4 & $<0.1$ & 2.7 & 0.9 & 0.1 & 7 & 0.1 & 0.1 & 1.7 & 91,9 & 7,0 & 1,1 & 0.7 \\
\hline ST16_2 & 5.6 & 5.5 & $<0.1$ & 0.4 & 0.3 & $<0.1$ & 0.3 & 1.31 & 2 & 21.5 & $<0.1$ & $<20$ & $<0.2$ & $<0.1$ & 1.1 & 0.4 & $<0.1$ & 13 & 0.2 & 0.1 & 2.5 & 84,0 & 14,4 & 1,6 & 6.1 \\
\hline ST16_3 & 5.5 & 3.9 & $<0.1$ & 0.2 & 0.2 & $<0.1$ & $<0.2$ & 0.9 & 1.3 & $<20$ & $<0.1$ & $<20$ & $<0.2$ & $<0.1$ & 0.7 & 0.3 & $<0.1$ & 36 & 0.2 & 0.1 & 2 & 85,5 & 10,7 & 3,8 & 4 \\
\hline FP9_0 & 5.7 & 20.5 & $<0.1$ & 1.2 & 0.6 & $<0.1$ & 5.5 & 0.2 & 3.7 & 112.2 & 1.2 & 106.2 & 0.9 & 0.4 & 1.6 & 0.1 & 0.3 & 5 & 1.5 & 0.5 & 2.9 & 83,8 & 11,1 & 5,1 & 14.9 \\
\hline FP9_1 & 5.6 & 10.1 & $<0.1$ & 0.9 & 0.4 & $<0.1$ & 1.1 & 0.6 & 3.2 & 134.9 & 0.4 & 133.3 & 0.5 & 0.1 & 1.1 & 0.2 & 0.4 & 101 & 0.6 & 0.1 & 5.5 & 46,3 & 34,2 & 19,5 & 12.6 \\
\hline FP9_2 & 5.5 & 8.2 & $<0.1$ & 0.7 & 0.2 & $<0.1$ & 0.9 & 0.6 & 1.7 & 99 & 0.3 & 95.6 & 0.4 & 0.1 & 0.9 & 0.2 & 0.3 & 0 & & & & & & & \\
\hline FP19_0 & 9.9 & 1046 & 2 & 182.7 & 113.7 & 0.3 & 2.9 & 1 & 186.4 & 879.4 & 0.5 & 1263.8 & 4.3 & 0.5 & 228.4 & 0.3 & 2.3 & 0 & 0.2 & 0.1 & 2.8 & 77,7 & 19,1 & 3,2 & 23.7 \\
\hline FP19_1 & 7.2 & 141.2 & 0.3 & 24 & 13.8 & 0.05 & 0.2 & 0.3 & 18.8 & 303.2 & 0.3 & 457.4 & 0.7 & 0.2 & 33.8 & $<0.1$ & 0.8 & 0 & 0.3 & 0.1 & 3.9 & 89,5 & 10,1 & 0,5 & 0.7 \\
\hline FP19_2 & 6.1 & 22.5 & 0.3 & 3.8 & 0.7 & $<0.1$ & $<0.2$ & 1.3 & 4.8 & 952.1 & 0.2 & 729.5 & 0.7 & 0.2 & 4.5 & 0.4 & 2.4 & 134 & 0.2 & 0.1 & 2.8 & 86,3 & 12,2 & 1,5 & 2.2 \\
\hline
\end{tabular}



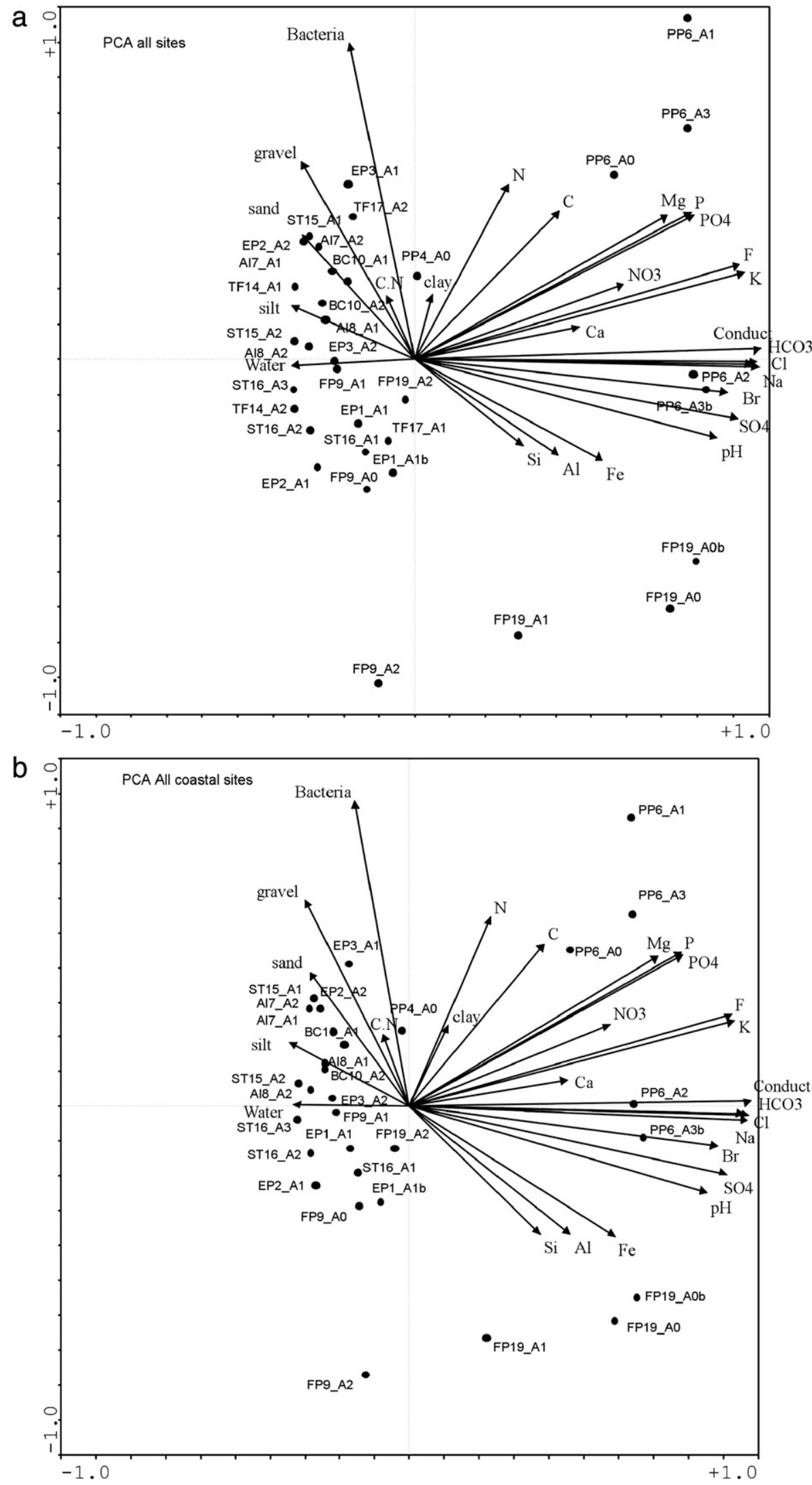

Fig. 2. a. Principal Component Analysis of all the investigated sites. See Table 2 for abbreviations. b. Principal Component Analysis of the coastal sites. See Table 2 for abbreviations. 
there is no modern penguin rookery nearby. The active layer is highly variable among as well as within sites. All the investigated soils are Gelisols (Soil Survey Staff, 2006) (Table 1). Most of them are Haplorthels (with one lithic haplorthels, EP3, one Acquic Haplorthels, EP2, and the others being Glacic Haplorthels), three are Psammorthels (EP1, TF17, FP19) and only one soil (AI7) is a Glacic Haploturbels.

The physical and chemical parameters of the soils change within as well as among sites (Table 2). Soil pH (4.99-9.92) ranges from very strongly acid to very strongly alkaline, with conductivity, $\mathrm{F}, \mathrm{Cl}$, sulfate, phosphate, $\mathrm{Al}$, and $\mathrm{Fe}$ also showing wide ranges of variation. Organic $\mathrm{C}$ and total $\mathrm{N}$ are generally very low. Grain size is coarse or very coarse in all sites, with the finer material (silt and clay) always less than $30 \%$ and mainly below $20 \%$. Water content is highly variable $(0.7 \%-30.3 \%)$.

The northernmost site at Apostrophe Island is on a flat icefree area close to a glacier boundary and is characterized by gabbro outcrops and morainic till, locally reworked by periglacial features such as unsorted patterned grounds and frost fissures polygons. Here two plots were selected: one (AI7) vegetated with macrolichens (Usnea sphacelata with total coverage more than $50 \%$ ) and one not vegetated (AI8), with active layer thicknesses of 43 and $45 \mathrm{~cm}$, respectively. The soil samples are characterized by acid values of $\mathrm{pH}$, low conductivity, coarse texture, and low water content, and they do not show extremely high or low values of the investigated parameters, which decrease with depth, with the exception of phosphate and $\mathrm{HCO}_{3}^{-}$.

Edmonson Point is a wide ice-free area on the eastern slope at the foot of Mount Melbourne, the active volcano close to the Mario Zucchelli Station (MZS). Here we selected three plots, one on bare ground (EP3), one vegetated by mosses in moist conditions (EP1), and one with mosses along a stream (EP2). EP3 is located on a weathered basaltic outcrop, while EP1 and $\mathrm{EP} 2$ are on alluvium. The active layer ranges from $41 \mathrm{~cm}$ (EP1) to $82 \mathrm{~cm}$ (EP2). Bacteria and water content show here the highest values recorded among all the investigated sites. Comparing the soils of this site, EP1 has the highest values of nitrate, organic $\mathrm{C}$ and water content, EP2 has the lowest conductivity, but highest $\mathrm{Al}$ and silt, while EP3 has the highest values of $\mathrm{Cl}$, sulfate, $\mathrm{Fe}$, and $\mathrm{Na}$ (which may be related to the absence of vegetation uptake), and the highest recorded values of bacteria.

Boulder Clay is an ice-free and flat area very close to MZS and the selected plot stands within the CALM grid site on till composed mainly of granitic boulders and pebbles and with scattered mosses and crustose lichens. The chemical and physical parameters of the soil do not show peculiar characteristics at this site, except for the relatively high values of $\mathrm{Al}$ and $\mathrm{Fe}$.

Tarn Flat is the only inland site, located on the largest ice-free area close to the MZS. It is characterised by till composed mainly of granitic boulders and pebbles, and the sampling sites are on flat areas with bare ground at different elevations (TF14 at $100 \mathrm{~m}$ a.s.1. and TF17 at $20 \mathrm{~m}$ a.s.1.). The active layer ranges from $42 \mathrm{~cm}$ (TF14) to $58 \mathrm{~cm}$ (TF17). Comparing the two soils, TF17 shows a strong enrichment of $\mathrm{HCO}_{3}^{-}, \mathrm{Al}$, and $\mathrm{Fe}$, and higher values of conductivity and $\mathrm{Na}$ than TF14. Both samples are characterised by very low values of nitrate, phosphate, organic $\mathrm{C}$ and total $\mathrm{N}$.

Prior Island in an ice-free island immediately south of the Drygalski ice tongue, which is characterized by till and the occurrence of ornithogenic soils. Here we selected two sampling sites at the top of the island (150 $\mathrm{m}$ a.s.l.), one with bare ground (PP6) and one with closed moss vegetation encrusted by lichens (PP4), with active layer thicknesses of 47 and $55 \mathrm{~cm}$, respectively. All the collected soil samples show very high conductivity and very low water content. PP6 is characterised by a very strong enrichment of all the analysed chemical parameters, with the highest values recorded in all the study area for $\mathrm{F}$, phosphate, $\mathrm{Mg}, \mathrm{P}$ and organic $\mathrm{C}$. The peculiar characteristics of PP6 are linked to its ornithogenic origin.

Starr Nunatak is a small, flat, granitic nunatak along the Harbord glacier and close to the coast where we investigated two plots, one with closed moss vegetation encrusted by lichens (ST15) and one on bare ground (ST16). The active layer thickness is deeper in the bare ground plot $(82 \mathrm{~cm})$ than in the vegetated one $(65 \mathrm{~cm})$. The soils are enriched in $\mathrm{Cl}$, sulfate,

Table 3

Eigenvalues and cumulative percentage variance of species data for the Principal Component Analysis carried out for all sites, for the coastal sites, for the coastal sites on granite lithology, for the unvegetated coastal sites and for the vegetated coastal sites

\begin{tabular}{lccccc}
\hline PCA all sites & \multicolumn{1}{l}{} \\
\hline Axes & 1 & 2 & 3 & 4 & $\begin{array}{l}\text { Total } \\
\text { variance }\end{array}$ \\
$\begin{array}{l}\text { Eigenvalues } \\
\begin{array}{l}\text { Cumulative percentage } \\
\text { variance of species data }\end{array}\end{array}$ & 0.482 & 0.196 & 0.111 & 0.1 & 1.000 \\
PCA coastal sites & 67.7 & 78.8 & 88.8 & \\
\hline Axes & 1 & 2 & 3 & 4 & $\begin{array}{l}\text { Total } \\
\text { variance }\end{array}$ \\
$\begin{array}{l}\text { Eigenvalues } \\
\begin{array}{l}\text { Cumulative percentage } \\
\text { variance of species data }\end{array}\end{array}$ & 0.491 & 0.2 & 0.108 & 0.095 & 1.000 \\
& & 79.9 & 89.4 & &
\end{tabular}

PCA coastal sites on granite lithology

\begin{tabular}{|c|c|c|c|c|c|}
\hline Axes & 1 & 2 & 3 & 4 & $\begin{array}{l}\text { Total } \\
\text { variance }\end{array}$ \\
\hline Eigenvalues & 0.504 & 0.219 & 0,101 & 0.89 & 1.000 \\
\hline $\begin{array}{l}\text { Cumulative percentage } \\
\text { variance of species data }\end{array}$ & 50.4 & 72.4 & 82.5 & 91.4 & \\
\hline
\end{tabular}

PCA unvegetated coastal sites

\begin{tabular}{|c|c|c|c|c|c|}
\hline Axes & 1 & 2 & 3 & 4 & $\begin{array}{l}\text { Total } \\
\text { variance }\end{array}$ \\
\hline Eigenvalues & 0.536 & 0.211 & 0.11 & 0.067 & 1.000 \\
\hline $\begin{array}{l}\text { Cumulative percentage } \\
\text { variance of species data }\end{array}$ & 53.6 & 74.4 & 85.7 & 92.4 & \\
\hline
\end{tabular}

PCA vegetated coastal sites

\begin{tabular}{|c|c|c|c|c|c|}
\hline Axes & 1 & 2 & 3 & 4 & $\begin{array}{l}\text { Total } \\
\text { variance }\end{array}$ \\
\hline Eigenvalues & 0.334 & 0.202 & 0.147 & 0.117 & 1.000 \\
\hline $\begin{array}{l}\text { Cumulative percentage } \\
\text { variance of species data }\end{array}$ & 33.4 & 53.5 & 68.2 & 79.9 & \\
\hline
\end{tabular}


Table 4

Pearson correlation matrix for all sites

\begin{tabular}{|c|c|c|c|c|c|c|c|c|c|c|c|c|c|c|c|c|c|c|c|c|c|c|c|c|c|c|}
\hline & $\mathrm{pH}$ & Conduc. & $\mathrm{F}^{-}$ & $\mathrm{Cl}^{-}$ & $\mathrm{SO}_{4}^{-}$ & $\mathrm{Br}^{-}$ & $\mathrm{NO}_{3}^{-}$ & $\mathrm{PO}_{4}^{-}$ & $\mathrm{HCO}^{-}$ & $\mathrm{Al}$ & $\mathrm{Ca}$ & $\mathrm{Fe}$ & K & $\mathrm{Mg}$ & $\mathrm{Na}$ & $\mathrm{P}$ & $\mathrm{Si}$ & Bacteria & org & $\mathrm{N}$ & $\mathrm{C} / \mathrm{N}$ & gravel & sand & silt & clay & Wat \\
\hline & 00 & & .17 & 87 & 0.85 & -0.75 & -0.09 & 0.41 & & 49 & 0.34 & 0.67 & -0.18 & 0.50 & 0.94 & 0.18 & -0.25 & -( & 32 & .30 & -0.01 & -0.09 & 0.06 & 0.07 & 0.03 & . \\
\hline & 0.93 & & & & 0.81 & .79 & -0.03 & 0.55 & & 33 & & 52 & .18 & & 95 & 0.08 & & 0. & & 0.4 & & 0.09 & -0.08 & & 00 & 0.05 \\
\hline & -0.17 & .13 & & 0.13 & -0.09 & 17 & 0.37 & -0.13 & -0.12 & -0.14 & 0.42 & -0.11 & 0.66 & 0.28 & -0.13 & -0.27 & 0.74 & -0.1 & 14 & -0.19 & -0.07 & 0.14 & -0.03 & -0.19 & -0.15 & -0.17 \\
\hline & 0.87 & 0.93 & & 1.00 & 0.87 & .71 & 0.04 & 0.28 & 0.84 & 0.42 & 0.33 & 0.62 & -0.17 & -0.44 & 0.95 & -0.03 & 19 & -0. & 40 & 0.24 & 0.1 & 0.06 & -0.06 & 0.00 & & 0.15 \\
\hline & & & & 87 & 1.00 & .50 & -0.11 & 0.05 & 0.74 & 0.56 & -0.23 & 0.79 & -0.13 & -0.34 & 0.94 & -0.08 & -0.13 & -0.0 & -0.01 & -0.05 & 0.01 & -0.16 & 0.12 & 0.13 & -0. & 0.30 \\
\hline & 0.75 & 0.79 & 17 & 0.71 & -0.50 & 00 & 0.02 & -0.53 & -0.76 & 0.12 & 0.46 & 0.27 & 0.23 & 0.62 & -0.70 & -0.15 & 0.27 & 0.02 & -0.65 & -0.42 & -0.34 & -0.03 & -0.06 & 0.19 & 0.07 & -0.07 \\
\hline & .09 & -0.03 & & & -0.11 & 02 & 00 & 03 & -0.0 & 22 & 0.07 & & 0.36 & .14 & -0.0 & 0.01 & 9 & 0. & 08 & -0.12 & 0.01 & -0.01 & 0.06 & -0 . & 0.11 & -0.30 \\
\hline & & & & & & 53 & 03 & & & .13 & 0.20 & -0.10 & 0.05 & -0.28 & & 16 & -0. & 0. & 64 & 0.60 & -0. & 0.20 & -0.16 & -0 . & 13 & -0.33 \\
\hline & & & & & & & & & & 29 & & & -0.17 & & & & & & & .48 & -0.01 & 0.11 & -0.10 & & & $0.0 c$ \\
\hline & & & & & & & & & & & & & & & & & & & & & & & & & & \\
\hline & 34 & -0 & & & & & & & & & & & & & & & & & & & & & & & & \\
\hline & & & & & & & & & & & & & & & & & & & & & & & 0.34 & & & 0.13 \\
\hline & & - & & & & & & & & & & & & & & & & & & & & 0.15 & -0.06 & & 6 & -0.22 \\
\hline & & & & & & & & & & & & 1 & & & & & & & & & & & 01 & & & -0.10 \\
\hline $\mathbf{a}$ & & & & & & & & & & & & & & & & & & & & & & & & & & 0.17 \\
\hline & & & & & - & & & & & & & & & & & & & & & & & & & & & $-0.0^{7}$ \\
\hline & - & -0.2 & & 9 & -0 . & & & -0.2 & -0.2 & & & 4 & & 39 & -0 . & -0. & & & & -0 & & & 0.06 & & -0. & -0.0 \\
\hline & -( & & & .01 & -0.09 & & & & & 5 & & -0.08 & -0.1 & -0.01 & -0.0 & & -0 . & & & 0.09 & -0.11 & -0. & 0.03 & & & -0.07 \\
\hline o & & & & & -0. & & & & & & & -0.2 & & & & & & & & 0. & & & -0. & & & -0.07 \\
\hline & & & & & -0.0 & & -0.1 & & & & & -0.2 & & & & & -0 . & & & 1.00 & -0 . & & -0.48 & & & -0.30 \\
\hline & - & & .07 & 0 & & -0.3 & & -0.09 & -0. & 3 & & 0.00 & -0.2 & -0.4 & 0.03 & -0. & 0.0 & & & -0.2 & 1. & 0. & 0.00 & & & 0.5 \\
\hline & & & & & -0.16 & -0.03 & -0.01 & & & 1 & -0.04 & -0.4 & & 0.06 & -0.04 & -0.2 & 0.1 & -0. & & 0. & 0.0 & 00 & -0.89 & & -0. & -0.1 \\
\hline nc & & -0.08 & .03 & -0.06 & 0.12 & -0.06 & 0.06 & -0.16 & -0.10 & 0.36 & & 0.34 & -0.06 & 0.01 & 0.03 & 0.13 & 0.06 & 0.03 & 0.40 & -0.48 & 0.00 & -0.89 & 1.00 & -0.03 & -0.23 & 0.07 \\
\hline & & & & & & & -0.19 & -0.22 & -0. & & & & & & & & & -0.1 & & -0.05 & -0.09 & -0.36 & -0.03 & & 0.03 & 0.3 \\
\hline & & & & & -0.0 & & & & & & & & & & -0.0 & & & & & & & -0.05 & -0.23 & .03 & 1.00 & -0.1 \\
\hline Water & 0.07 & 0.05 & -0.17 & 0.15 & 0.30 & -0.07 & -0.30 & -0.33 & 0.00 & 0.05 & 0.00 & 0.13 & -0.22 & -0.10 & 0.17 & -0.07 & -0.07 & -0.07 & -0.07 & -0.30 & $\mathbf{0 . 5 0}$ & -0.14 & 0.07 & 0.32 & -0.18 & 1.0 \\
\hline
\end{tabular}

In bold significant correlations with $\mathrm{p}<0.05$. 
phosphate, $\mathrm{HCO}_{3}^{-}$and $\mathrm{Na}$ in the bare ground plot (ST16) with respect to the vegetated one (ST15), which shows higher nitrate and organic $\mathrm{C}$ concentrations in the upper soil layers.

Finger Point is the southernmost location along the latitudinal gradient and is a flat area along the coast characterized by till deposits mainly composed of granitic boulders. Also in this case, we selected two sites close to each other but with different vegetation (FP9 with closed moss cover, and FP19 on bare ground). Also at Finger Point, the bare ground has a thicker active layer than the moss vegetated ground (73 versus $62 \mathrm{~cm}$ ). The two soils show very different characteristics; moreover, FP19 is totally different from all the other analysed soils with the highest values of most of the investigated parameters $(\mathrm{pH}$, conductivity, $\mathrm{Cl}$, sulfate, $\mathrm{HCO}_{3}^{-}, \mathrm{Al}, \mathrm{Fe}, \mathrm{Na}, \mathrm{Si}$ ).

\subsection{Patterns of soil characteristics with latitude}

To investigate the potential occurrence of broad patterns influencing the soil characteristics, two Principal Component Analyses (PCA) were performed, one considering all the investigated sites (Fig. 2a; Table 3) and the other only the coastal sites (Fig. 2b; Table 3). PCA provides indirect information on the relations between the analysed parameters (represented by vectors/arrows) and the sites (represented by points). The length of the vectors/arrows indicates the importance of the analysed parameters, the distribution of the vectors indicates a positive or negative influence respect to the clusters of sites.
The two analyses clearly indicate that the broad and general patterns of soil characteristics do not exhibit consistent variation with regard to site location (coast versus inland, as shown in Fig. 2a), latitude, lithology and vegetation (Fig. 2b). Both analyses discriminate from all the other samples the ornithogenic soil (PP6) and the samples with very high $\mathrm{Fe}, \mathrm{Al}$ and $\mathrm{Si}$ concentrations (FP19) independently of their depth.

Considering the soil chemical parameters, some relations indicated by PCA were similar to those outlined by the Pearson correlation matrix for all sites (Table 4), which allowed us to identify groups of correlated parameters. In particular, positive correlations were observed for: 1) $\mathrm{pH}$, conductivity, $\mathrm{Cl}, \mathrm{Na}$, sulfate and $\left.\mathrm{HCO}_{3}^{-}, 2\right) \mathrm{Al}$ and $\mathrm{Fe}, 3$ ) organic carbon and total nitrogen. Sand and gravel are inversely correlated (when the first increases the latter decreases and vice versa). Unlike the Pearson correlation matrix, both PCA (Fig. 2a and 2b) indicated relations between $\mathrm{K}$ and $\mathrm{F}$ and between $\mathrm{P}$ and $\mathrm{PO} 4$ along the first axis, although along the second axis, all the grain size components were positively related.

To assess the influence of latitude avoiding potential interference linked to the different lithology, a third PCA (Fig. 3; Table 3) was performed considering only the coastal sites with granitic lithology. Unfortunately the latitudinal gradient referring to these sites was more limited $\left(74^{\circ}-77^{\circ} \mathrm{S}\right)$ with respect to the whole investigated transect. Nevertheless, PCA showed there was no appreciable pattern with latitude and that the patterns of soil parameters were very similar to the above-

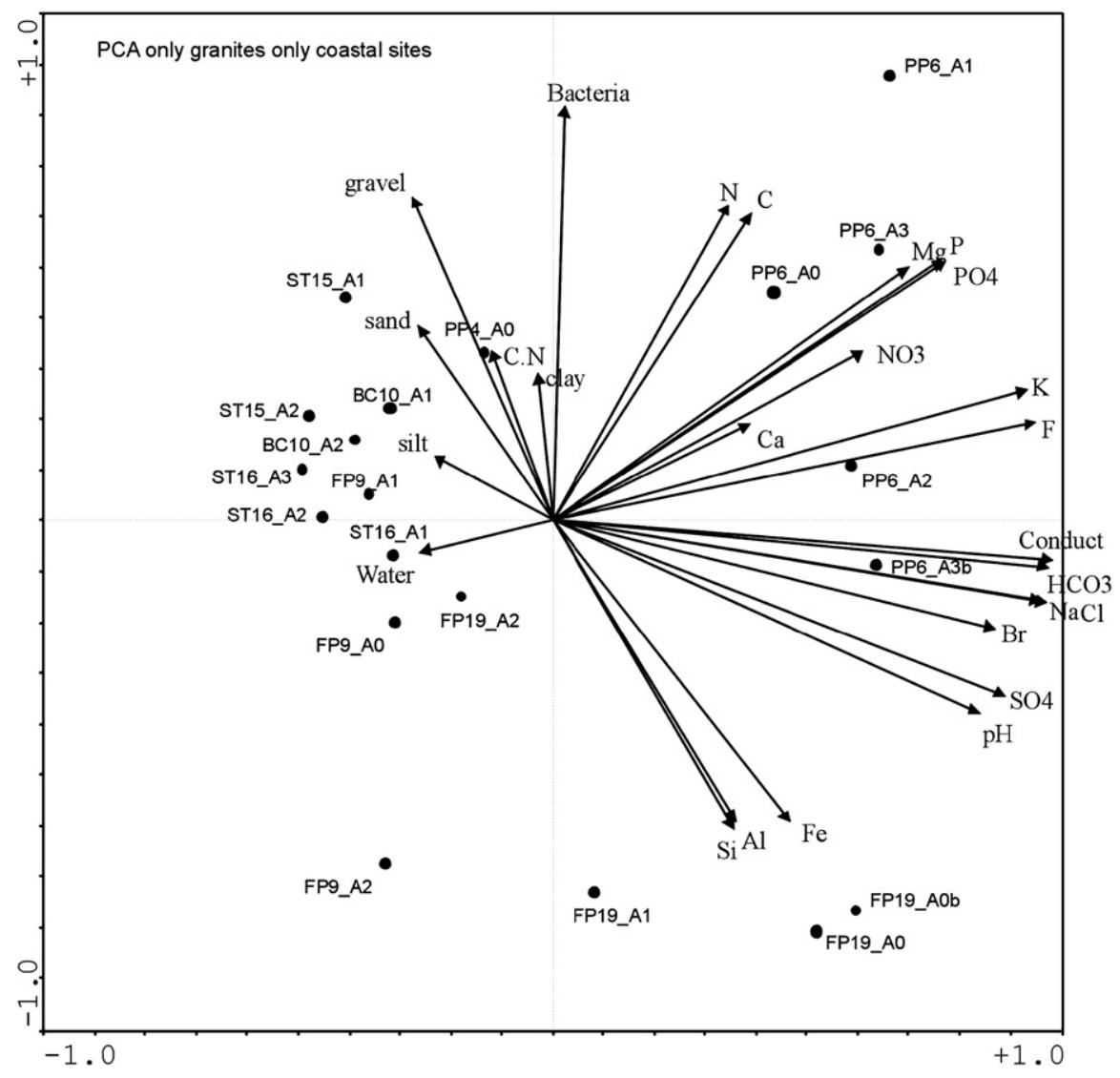

Fig. 3. Principal Component Analysis of the coastal sites with granitic lithology. See Table 2 for abbreviations. 
mentioned results. Also by eliminating the samples with peculiar characteristics (PP6 and FP19), the PCA provided similar results (data not shown).

Further, to avoid the potential influence of vegetation, we performed the PCA considering only the unvegetated coastal sites (Fig. 4; Table 3). Also in this case, latitude did not exert any significant influence on soil characteristics and the soil parameters showed the same patterns already outlined in Figs. 2a, b and 3.

The Pearson correlation matrix of the unvegetated sites (Table 5) confirms the general patterns obtained for all sites (Table 4), in particular concerning 1) the link between $\mathrm{pH}$, conductivity, $\mathrm{Na}, \mathrm{Cl}$, sulfate, and $\mathrm{HCO}_{3}^{-}, 2$ ) between $\mathrm{Al}$ and $\mathrm{Fe}$, and 3 ) the negative relation between gravel and sand. In addition, the unvegetated sites are characterized by the following positive correlations: 1) $\mathrm{F}$ and $\mathrm{K}$ (shown also by the PCA); 2) total nitrogen and phosphate; 3) organic carbon and total nitrogen.

\subsection{Patterns of soil characteristics with lithology}

From qualitative observations of the chemical and physical data, the samples of both Apostrophe Island (gabbro) and Edmonson Point (basalts) (Table 2) are characterized by slightly lower contents of $\mathrm{Al}, \mathrm{Fe}$ and $\mathrm{Ca}$, and $\mathrm{K}$ than the samples collected in the granitic sites. In addition, PCA indicates that lithology does not influence soil properties, when considering all the investigated sites (Fig. 2a), only the coastal sites (Fig. 2b), and the unvegetated coastal sites as well (Fig. 4).

\subsection{Patterns of soil characteristics with vegetation}

The influence of vegetation occurrence was assessed by comparing the surface layers of vegetated soils with those of bare ground at each site (i.e. AI7_1 vs AI8_1, EP1_1 vs EP3_1, ST15_1 vs ST16_1, FP9_1 vs FP19_1) (Table 2). In most cases, the vegetated surface layers show lower values of $\mathrm{pH}$, anions (except for Apostrophe Island samples) and cations, but higher values of nitrate, organic $\mathrm{C}$, total $\mathrm{N}$, and water. Moreover, in the vegetated soils the values of the chemical parameters tend mainly to decrease with depth.

The PCA of the vegetated sites (Fig. 5; Table 3) indicates that there are no evident patterns with respect to vegetation type, although there is a sorting which separates the surface from the deeper layers. In fact, most of the surface layers show higher values of organic $\mathrm{C}$, total $\mathrm{N}$, and water, and a higher $\mathrm{C} / \mathrm{N}$ ratio. The enrichment of organic $\mathrm{C}$, total $\mathrm{N}$ and water of the surface layers with respect to the deeper layers is confirmed also by the values reported in Table 2 and is an effect clearly linked to vegetation occurrence, independently of site location and lithology.

The Pearson correlation matrix of the vegetated sites (Table 6) shows different relations between the analyzed parameters and those obtained considering all sites and only

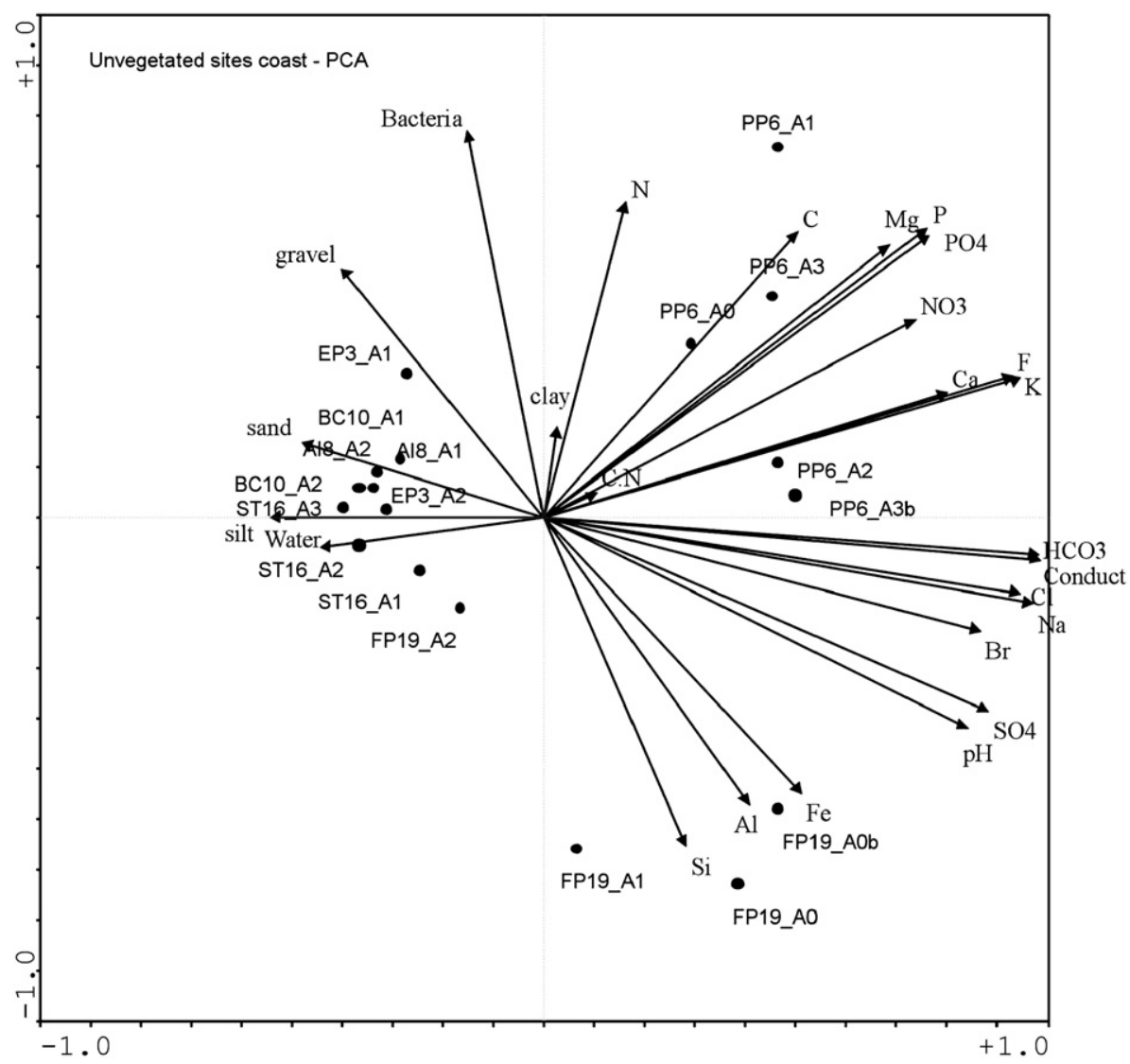

Fig. 4. Principal Component Analysis of the unvegetated coastal sites. See Table 2 for abbreviations. 
Table 5

Pearson correlation matrix referred only to the vegetated sites

\begin{tabular}{|c|c|c|c|c|c|c|c|c|c|c|c|c|c|c|c|c|c|c|c|c|c|c|c|c|c|c|}
\hline & $\mathrm{pH}$ & Conduc. & $\mathrm{F}^{-}$ & $\mathrm{Cl}^{-}$ & $\mathrm{SO}_{4}^{-}$ & $\mathrm{Br}^{-}$ & $\mathrm{NO}_{3}^{-}$ & $\mathrm{PO}_{4}^{-}$ & $\mathrm{HCO}^{-}$ & $\mathrm{Al}$ & $\mathrm{Ca}$ & $\mathrm{Fe}$ & K & $\mathrm{Mg}$ & $\mathrm{Na}$ & $\mathrm{P}$ & $\mathrm{Si}$ & Bacteria & Corg & $\mathrm{N}$ & $\mathrm{C} / \mathrm{N}$ & Gravel & Sand & Silt & Clay & Water \\
\hline PH & 1.00 & 0.08 & -0.22 & -0.04 & -0.09 & -0.04 & -0.12 & -0.13 & 0.25 & 0.30 & -0.04 & 0.21 & 0.06 & -0.03 & 0.26 & 0.30 & -0.53 & -0.01 & -0.14 & 0.41 & -0.36 & -0.04 & -0.19 & 0.24 & 0.29 & 0.13 \\
\hline Conductivity & 0.08 & 1.00 & -0.37 & 0.69 & 0.70 & -0.57 & -0.28 & -0.14 & 0.53 & 0.33 & -0.43 & 0.30 & -0.64 & -0.70 & 0.83 & -0.21 & -0.37 & 0.13 & 0.58 & 0.10 & 0.35 & -0.16 & 0.09 & -0.06 & 0.31 & 0.40 \\
\hline $\mathbf{F}^{-}$ & -0.22 & -0.37 & 1.00 & -0.32 & -0.39 & 0.07 & 0.54 & -0.12 & -0.11 & -0.04 & 0.29 & -0.03 & 0.45 & 0.17 & -0.36 & -0.20 & 0.68 & -0.12 & -0.12 & -0.19 & 0.01 & -0.06 & 0.27 & -0.26 & -0.16 & -0.22 \\
\hline $\mathrm{Cl}^{-}$ & -0.04 & 0.69 & -0.32 & 1.00 & 0.80 & -0.10 & -0.51 & -0.01 & 0.30 & 0.25 & -0.15 & 0.26 & -0.62 & -0.33 & 0.68 & -0.33 & -0.36 & -0.20 & 0.16 & 0.07 & 0.13 & -0.12 & -0.05 & 0.21 & 0.19 & 0.10 \\
\hline $\mathrm{SO}_{4}^{-}$ & -0.09 & 0.70 & -0.39 & 0.80 & 1.00 & -0.41 & -0.38 & 0.11 & 0.12 & 0.06 & -0.09 & -0.01 & -0.39 & -0.29 & 0.66 & -0.21 & -0.54 & -0.08 & 0.19 & 0.03 & 0.14 & -0.18 & 0.10 & 0.15 & 0.03 & 0.23 \\
\hline $\mathrm{Br}^{-}$ & -0.04 & -0.57 & 0.07 & -0.10 & -0.41 & 1.00 & 0.09 & 0.13 & 0.00 & 0.18 & 0.23 & 0.20 & 0.15 & 0.38 & -0.45 & 0.20 & 0.10 & 0.14 & -0.63 & 0.06 & -0.59 & 0.11 & -0.28 & 0.19 & 0.15 & -0.66 \\
\hline $\mathrm{NO}_{3}^{-}$ & -0.12 & -0.28 & 0.54 & 0.51 & -0.38 & 0.09 & 1.00 & 0.17 & 0.24 & 0.17 & 0.21 & 0.11 & 0.45 & -0.04 & -0.25 & 0.27 & 0.31 & 0.42 & -0.25 & -0.36 & -0.09 & -0.19 & 0.27 & -0.24 & 0.17 & -0.28 \\
\hline $\mathrm{PO}_{4}^{-}$ & -0.13 & -0.14 & -0.12 & -0.01 & 0.11 & 0.13 & 0.17 & 1.00 & 0.04 & 0.23 & 0.22 & 0.27 & 0.46 & -0.02 & 0.07 & 0.62 & -0.17 & -0.17 & -0.33 & -0.39 & -0.16 & -0.30 & 0.34 & -0.16 & 0.20 & -0.18 \\
\hline $\mathrm{HCO3}^{-}$ & 0.25 & 0.53 & -0.11 & 0.30 & 0.12 & 0.00 & 0.24 & 0.04 & 1.00 & 0.88 & -0.35 & 0.84 & -0.37 & -0.70 & 0.63 & 0.36 & -0.23 & 0.58 & -0.02 & -0.23 & 0.10 & -0.30 & -0.02 & 0.04 & 0.87 & 0.00 \\
\hline Al & 0.30 & 0.33 & -0.04 & 0.25 & 0.06 & 0.18 & 0.17 & 0.23 & 0.88 & 1.00 & -0.37 & 0.97 & -0.08 & -0.5 & 0.55 & & -0.16 & & -0.25 & -0.16 & -0.15 & -0.2 & -0.04 & -0.03 & 0.97 & -0.27 \\
\hline $\mathrm{Ca}$ & -0.04 & -0.43 & 0.29 & -0.15 & -0.09 & 0.23 & 0.21 & 0.22 & -0.35 & -0.37 & 1.00 & -0.38 & 0.36 & 0.59 & -0.24 & 0.10 & 0.04 & -0.1 & -0.39 & -0.39 & -0.07 & -0.28 & 0.47 & 0.03 & -0.48 & -0.06 \\
\hline $\mathrm{Fe}$ & 0.21 & 0.30 & -0.03 & 0.26 & -0.01 & 0.20 & 0.11 & 0.27 & 0.84 & 0.97 & -0.38 & 1.00 & -0.09 & -0.52 & 0.51 & 0.44 & -0.05 & 0.39 & -0.20 & -0.20 & -0.08 & -0.23 & -0.04 & -0.12 & 0.94 & -0.29 \\
\hline K & 0.06 & -0.64 & 0.45 & -0.62 & -0.39 & 0.15 & 0.45 & 0.46 & -0.37 & -0.08 & 0.36 & -0.09 & 1.00 & 0.38 & -0.51 & 0.45 & 0.21 & -0.1 & & -0.18 & -0.32 & & 21 & -0.28 & -0.09 & -0.28 \\
\hline Ig & -0.03 & -0.70 & & -0.33 & -0.29 & 0.38 & -0.04 & -0.02 & -0.70 & -0.55 & 0.59 & -0.52 & 0.38 & 1.00 & -0.48 & -0.03 & 0.25 & -0.21 & -0.52 & 0.19 & -0.48 & -0. & 26 & 0.21 & -0.58 & -0.36 \\
\hline $\mathrm{Ja}$ & 0. & 0.83 & 6 & 0.68 & 0.66 & -0.45 & -0.25 & 0.07 & 0.63 & 0.55 & -0.24 & 0.51 & 0.51 & -0.48 & 1.00 & 0.08 & -0.43 & 0.19 & 13 & -0.01 & 0.16 & -0.48 & 0.29 & 0.09 & 0.50 & 0.21 \\
\hline & 0.30 & -0.21 & -0.20 & .33 & 0.21 & 0.20 & 0.27 & 0.62 & 0.36 & 0.48 & 0.10 & 0.44 & 0.45 & -0.03 & 0.08 & 1.00 & -0.29 & 0.26 & -0.42 & -0.35 & -0.20 & -0.49 & 0.23 & 0.21 & 0.52 & 0.00 \\
\hline i & -0.53 & -0.37 & 0.68 & -0.36 & -0.54 & 0.10 & 0.31 & -0.17 & -0.23 & -0.16 & 0.04 & -0.05 & 0.21 & 0.25 & -0.43 & -0.29 & 1.00 & -0.04 & 0.10 & -0.19 & 0.17 & 0.02 & 0.29 & -0.42 & -0.22 & -0.29 \\
\hline Sacter & -0.01 & 0.13 & .12 & -0.20 & -0.08 & 0.14 & 0.42 & -0.17 & 0.58 & 0.46 & -0.12 & 0.39 & -0.15 & -0.21 & 0.19 & 0.26 & -0.04 & 1.00 & -0.22 & -0.19 & -0.08 & -0.13 & 0.02 & -0.14 & 0.51 & -0.32 \\
\hline Cor: & -0.14 & 0.58 & .12 & 0.16 & 0.19 & -0.63 & -0.25 & -0.33 & -0.02 & -0.25 & -0.39 & -0.20 & -0.37 & -0.52 & 0.13 & -0.42 & 0.10 & -0.22 & 1.00 & 0.04 & 0.66 & 0.27 & -0.06 & -0.29 & -0.23 & 0.66 \\
\hline & 0.41 & 0. & -0.19 & 0.07 & 0.03 & 0.06 & .36 & .39 & -0.23 & -0.16 & -0.39 & -0.20 & -0.18 & 0.19 & -0.01 & -0.35 & -0.19 & -0.19 & 0.04 & 1.00 & -0.61 & 0.26 & -0.32 & 0.18 & -0.16 & -0.18 \\
\hline $\mathrm{C} / \mathrm{N}$ & -0.36 & 0.35 & 0.01 & 0.13 & 0.14 & -0.59 & -0.09 & -0.16 & 0.10 & -0.15 & -0.07 & -0.08 & -0.32 & -0.48 & 0.16 & -0.20 & 0.17 & -0.08 & 0.66 & -0.61 & 1.00 & 0.05 & 0.06 & -0.15 & -0.11 & 0.70 \\
\hline Gravel & -0.04 & -0.16 & -0.06 & -0.12 & -0.18 & 0.11 & -0.19 & -0.30 & -0.30 & -0.29 & -0.28 & -0.23 & -0.03 & -0.14 & -0.48 & -0.49 & 0.02 & -0.13 & 0.27 & 0.26 & 0.05 & 1.00 & -0.81 & -0.39 & -0.20 & -0.11 \\
\hline Sand & -0.19 & 0.09 & 0.27 & -0.05 & 0.10 & -0.28 & 0.27 & & -0.02 & -0.04 & 0.47 & -0.04 & 0.21 & 0.26 & 0.29 & 0.23 & 0.29 & 0.02 & -0.06 & -0.32 & 0.06 & -0.81 & 1.00 & -0.10 & -0.16 & 0.05 \\
\hline Silt & 0.24 & -0.06 & -0.26 & 0.21 & 0.15 & 0.19 & -0.24 & -0.16 & 0.04 & -0.03 & 0.03 & -0.12 & -0.28 & 0.21 & 0.09 & 0.21 & -0.42 & -0.14 & -0.29 & 0.18 & -0.15 & -0.39 & -0.10 & 1.00 & 0.01 & 0.29 \\
\hline Clay & 0.29 & 0.31 & & & 0.03 & 0.15 & 0.17 & & 0.87 & 0.97 & -0.48 & 0.94 & -0.09 & -0.58 & 0.50 & 0.52 & -0.22 & 0.51 & -0.23 & -0.16 & -0.11 & -0.20 & -0.16 & 0.01 & 1.00 & -0.23 \\
\hline Water & 0.13 & 0.40 & -0.22 & 0.10 & 0.23 & -0.66 & -0.28 & -0.18 & 0.00 & -0.27 & -0.06 & -0.29 & -0.28 & -0.36 & 0.21 & 0.00 & -0.29 & -0.32 & 0.66 & -0.18 & 0.70 & -0.11 & 0.05 & 0.29 & -0.23 & 1.00 \\
\hline
\end{tabular}

In bold significant correlations with $\mathrm{p}<0.05$. 


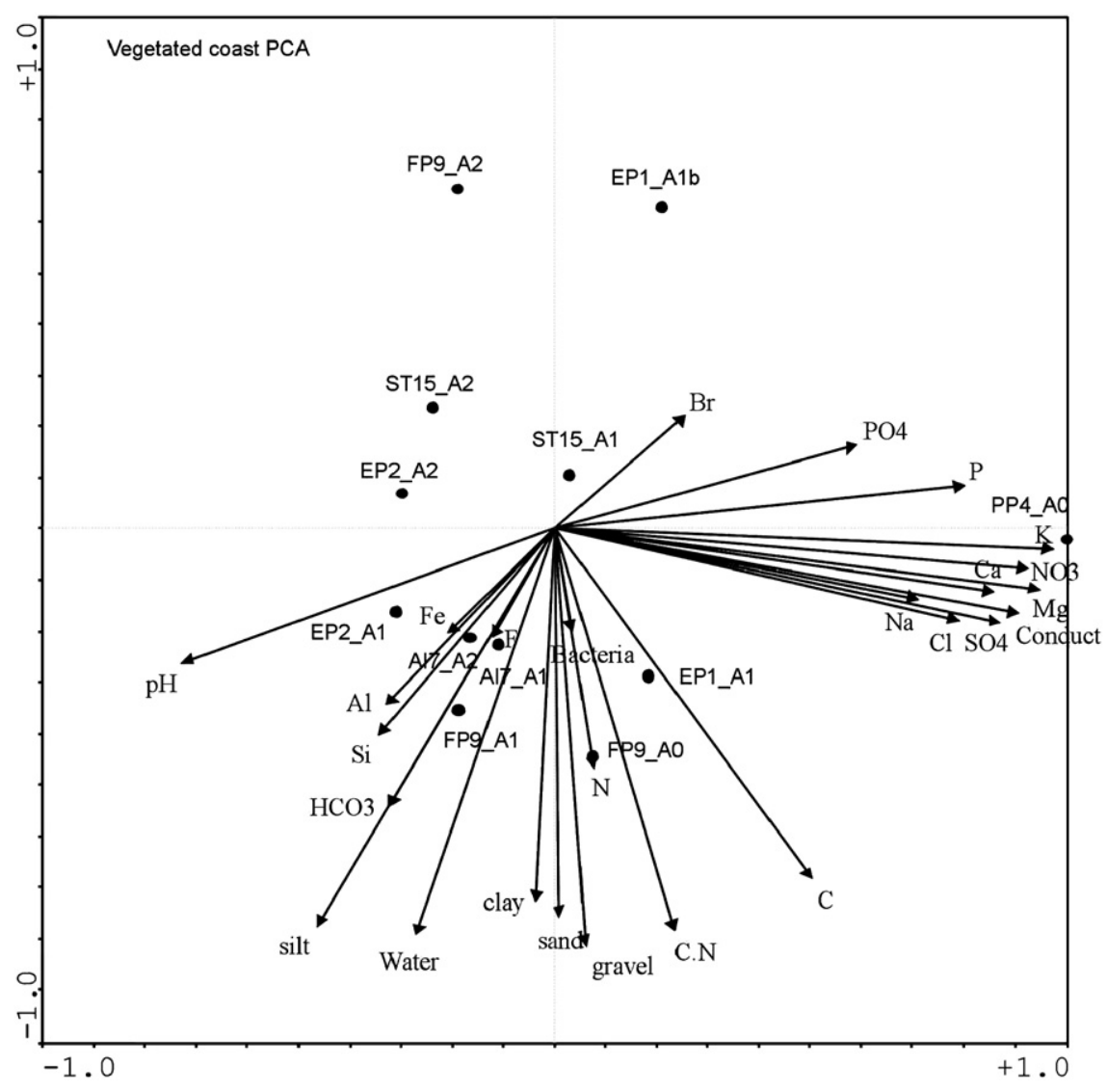

Fig. 5. Principal Component Analysis of the vegetated sites. See Table 2 for abbreviations.

the unvegetated ones, respectively. In particular: 1) there is a positive correlation between conductivity, $\mathrm{Na}$ and sulfate, which are all negatively related to $\mathrm{Mg}$; 2) there are positive correlations between $\mathrm{HCO}_{3}^{-}, \mathrm{Al}, \mathrm{Fe}$ and clay; 3 ) organic carbon, the $\mathrm{C} / \mathrm{N}$ ratio and water are positively linked to each other and all are negatively related to $\mathrm{Br} ; 4$ ) there is an inverse correlation between gravel and sand.

Both PCA and the correlation matrix demonstrate that vegetation occurrence influences the relationship between the chemical and physical properties of the soil. Moreover, the different vegetation types (moss vegetation, lichenized mosses vegetation, macrolichen vegetation, scattered epilithic vegetation with mosses) seem able to produce some differences in the chemical and/or physical characteristics of the investigated soils.

In particular, nitrate, organic carbon, the $\mathrm{C} / \mathrm{N}$ ratio and water content (Fig. 6) show different patterns depending on the vegetation type. Mosses have the highest water content and organic $\mathrm{C}$, and high values of $\mathrm{C} / \mathrm{N}$. Instead, mosses encrusted by lichens have the most xeric conditions, while their $\mathrm{C} / \mathrm{N}$ is the highest. The scattered vegetation shows the highest $\mathrm{N}$, the lowest $\mathrm{C}$ org and $\mathrm{C} / \mathrm{N}$, and low values of water. Also the activelayer thickness is influenced by vegetation type: in fact, the active layer is thinner in the moister sites (showing an inverse relation with the water content) and, therefore, it shows its lowest value under the moss vegetation (Fig. 6).

\section{Discussion}

Although climate along the latitudinal gradient and lithology are not significant sources of variation for soil properties, vegetation seems to influence the soil surface layer. However, the occurrence of ornithogenic soils exerts the highest influence on soil properties. These results are supported by the different PCA, carried out considering all sites, only the coastal sites, only the coastal unvegetated sites and only the coastal vegetated sites, respectively.

These results are compatible with previous studies that show no significant patterns of vegetation distribution (Cannone, 2006) and active layer thickness (Guglielmin, 2006) with latitude along the Ross Sea coast. Local microclimate appears to have a greater influence on site conditions (aspect, slope, altitude, distance from the sea) than regional climate along the environmental gradients. Microclimate influences the active layer, the thermal regime of the soil and water availability through modifying snow accumulation and persistence, wind speed at the ground and radiation balance. The importance of local scale environmental variability is confirmed by the occurrence of "hot spots" of biodiversity, irrespective of the latitudinal gradient, linked to the availability of favourable ecological niches for vegetation colonization and development.

In polar environments of the Northern Hemisphere, vegetation and active-layer and soil properties have for a long time 
Table 6

Pearson correlation matrix referred only to the unvegetated sites

\begin{tabular}{|c|c|c|c|c|c|c|c|c|c|c|c|c|c|c|c|c|c|c|c|c|c|c|c|c|c|c|}
\hline & $\mathrm{pH}$ & Conduc. & $\mathrm{F}^{-}$ & $\mathrm{Cl}^{-}$ & $\mathrm{SO}_{4}^{-}$ & $\mathrm{Br}^{-}$ & $\mathrm{NO}_{3}^{-}$ & $\mathrm{PO}_{4}^{-}$ & $\mathrm{HCO}^{-}$ & $\mathrm{Al}$ & $\mathrm{Ca}$ & $\mathrm{Fe}$ & $\mathrm{K}$ & $\mathrm{Mg}$ & $\mathrm{Na}$ & $\mathrm{P}$ & $\mathrm{Si}$ & Bacteria & Corg & $\mathrm{N}$ & $\mathrm{C} / \mathrm{N}$ & Gravel & Sand & Silt & Clay & Water \\
\hline & 1.00 & 0.92 & -0.36 & 0.85 & 0.86 & -0.78 & -0.22 & 0.37 & 0.90 & 0.49 & -0.53 & 0.68 & -0.33 & -0.72 & 0.94 & 0.27 & -0.43 & -0.22 & 0.25 & 0.30 & 0.18 & -0.08 & -0.07 & 0.50 & 0.41 & 0.22 \\
\hline $\begin{array}{c}\text { conductivity } \\
(\mu \mathrm{s} / \mathrm{cm})\end{array}$ & 0.92 & 1.00 & -0.35 & 0.91 & 0.79 & -0.82 & -0.15 & 0.54 & 0.97 & 0.29 & -0.59 & 0.49 & -0.30 & -0.65 & 0.94 & 0.17 & -0.48 & 0.00 & 0.48 & 0.51 & 0.16 & 0.22 & -0.34 & 0.28 & 0.49 & 0.22 \\
\hline fluoride & -0.36 & -0.35 & 1.00 & -0.31 & -0.21 & 0.42 & 0.22 & -0.23 & -0.31 & -0.25 & 0.62 & -0.22 & 0.99 & 0.50 & -0.31 & -0.38 & 0.77 & -0.22 & -0.24 & -0.23 & -0.24 & 0.32 & -0.33 & -0.01 & -0.06 & -0.09 \\
\hline chloride & 0.85 & 0.91 & -0.31 & 1.00 & 0.86 & -0.70 & -0.02 & 0.19 & 0.78 & 0.41 & -0.51 & 0.62 & -0.28 & -0.60 & 0.94 & -0.05 & -0.41 & -0.09 & 0.34 & 0.26 & 0.38 & 0.15 & -0.28 & 0.38 & 0.32 & 0.40 \\
\hline sulfate & 0.86 & 0.79 & -0.21 & 0.86 & 1.00 & -0.46 & -0.24 & -0.05 & 0.70 & 0.60 & -0.33 & 0.83 & -0.20 & -0.44 & 0.94 & -0.13 & -0.26 & -0.16 & -0.12 & -0.10 & 0.04 & -0.19 & 0.05 & 0.58 & 0.19 & 0.59 \\
\hline bromide & -0.78 & -0.82 & 0.42 & -0.70 & -0.46 & 1.00 & 0.10 & -0.62 & -0.77 & -0.06 & 0.71 & -0.24 & 0.35 & 0.80 & -0.69 & -0.48 & 0.58 & 0.05 & -0.62 & -0.61 & -0.45 & -0.18 & 0.25 & -0.07 & -0.49 & 0.17 \\
\hline trate & -0.22 & -0.15 & 0.22 & -0.02 & -0.24 & 0.10 & 1.00 & -0.09 & -0.22 & 0.24 & -0.08 & 0.02 & 0.24 & -0.21 & -0.18 & -0.36 & 0.06 & -0.18 & 0.17 & 0.03 & 0.29 & 0.19 & -0.21 & -0.02 & 0.10 & -0.31 \\
\hline phosphate & 0.37 & 0.54 & -0.23 & 0.19 & -0.05 & -0.62 & -0.09 & 1.00 & 0.67 & -0.30 & -0.43 & -0.29 & -0.17 & -0.38 & 0.25 & 0.52 & -0.36 & 0.24 & 0.77 & 0.90 & -0.08 & 0.48 & -0.51 & -0.25 & 0.50 & -0.44 \\
\hline HCO3- & 0.90 & 0.97 & .31 & 0.78 & 0.70 & -0.77 & -0.22 & 0.67 & 1.00 & 0.23 & -0.5 & 0.40 & -0.27 & -0. & 0.87 & 0.25 & -0 & 0 & 0.51 & 0.60 & 0.01 & 0.24 & -0.36 & 0.25 & 0.52 & 0.13 \\
\hline Al & 0.49 & 0.29 & -0.25 & 0.41 & 0.60 & -0.06 & 0.24 & -0.30 & 0.23 & 1.00 & -0.37 & 0.93 & -0.27 & -0.54 & 0.47 & -0.26 & -0.26 & -0.27 & -0.33 & -0.34 & 0.11 & -0.64 & 0.50 & 0.77 & 0.00 & 0.31 \\
\hline Ca & -0.53 & -0.59 & 0.62 & -0.51 & -0.33 & 0.71 & -0.08 & -0.43 & -0.55 & -0.37 & 1.00 & -0.38 & 0.58 & 0.82 & -0.50 & -0.20 & 0.81 & -0.36 & -0.43 & -0.42 & -0.32 & 0.22 & -0.17 & -0.12 & -0.31 & 0.06 \\
\hline $\mathrm{Fe}$ & 0.68 & 0.49 & -0.22 & 0.62 & 0.83 & -0.24 & 0.02 & -0.29 & 0.40 & & -0.38 & .00 & -0.24 & -0.55 & 0.70 & -0.21 & -0.26 & -0.2 & -0.34 & -0.34 & 0.11 & -0.58 & 0.43 & 0.81 & 0.02 & 0.47 \\
\hline K & -0.33 & -0.30 & 0.99 & -0.28 & -0.20 & 0.35 & 0.24 & -0.17 & -0.27 & -0.27 & 0.58 & -0.24 & 1.00 & 0.46 & -0.27 & -0.36 & 0.74 & -0.22 & -0.18 & -0.17 & -0.23 & 0.38 & -0.39 & -0.04 & -0.02 & -0.13 \\
\hline Mg & -0.72 & -0.65 & 0.50 & -0.60 & -0.44 & 0.80 & -0.21 & -0.38 & -0.59 & -0.54 & 0.82 & -0.55 & 0.46 & 1.00 & -0.60 & -0.27 & 0.66 & 0.20 & -0.37 & -0.35 & -0.47 & 0.23 & -0.13 & -0.33 & -0.43 & 0.21 \\
\hline $\mathrm{Na}$ & $\mathbf{0}$ & 0.94 & .31 & 0.94 & 0.94 & 0.69 & -0.18 & 0.25 & 0.87 & 0.47 & -0.50 & 0.70 & -0.27 & -0.60 & 1.00 & 0.02 & -0.41 & -0.10 & 0.18 & 0.19 & 0.13 & 0.00 & -0.14 & 0.44 & 0.35 & 0.42 \\
\hline $\mathbf{P}$ & 0.27 & 0.17 & -0.38 & -0.05 & -0.13 & -0.48 & -0.36 & 0.52 & 0.25 & -0.26 & -0.20 & -0.21 & -0.36 & -0.27 & 0.02 & 1.00 & -0.05 & -0.02 & 0.35 & 0.43 & 0.25 & -0.03 & 0.03 & -0.07 & 0.10 & -0.20 \\
\hline Si & -0.43 & -0.48 & 0.77 & -0.41 & -0.26 & 0.58 & 0.06 & -0.36 & -0.45 & -0.26 & 0.81 & -0.26 & 0.74 & 0.66 & -0.41 & -0.05 & 1.00 & -0.30 & -0.35 & -0.35 & -0.13 & 0.20 & -0.19 & 0.00 & -0.22 & 0.21 \\
\hline Bacteria & -0.22 & 0.00 & .22 & -0.09 & -0.16 & 0.05 & -0.18 & 0.24 & 0.05 & -0.27 & -0.36 & -0.27 & -0.22 & 0.20 & -0.10 & -0.02 & -0.30 & 1.00 & 0.18 & 0.21 & -0.22 & 0.08 & 0.00 & -0.21 & -0.33 & 0.18 \\
\hline Corg & 0.25 & 0.48 & .24 & 0.34 & -0.12 & -0.62 & 0.17 & 0.77 & 0.51 & -0.33 & -0.43 & -0.34 & -0.18 & -0.37 & 0.18 & 0.35 & -0.35 & 0.18 & 1.00 & 0.95 & 0.44 & 0.69 & -0.70 & -0.30 & 0.47 & -0.38 \\
\hline $\mathbf{N}$ & 0.30 & 0.51 & -0.23 & 0.26 & -0.10 & -0.61 & 0.03 & 0.90 & 0.60 & -0.34 & -0.42 & -0.34 & -0.17 & -0.35 & 0.19 & 0.43 & -0.35 & 0.21 & 0.95 & 1.00 & 0.20 & 0.63 & -0.66 & -0.31 & 0.55 & -0.42 \\
\hline $\mathrm{C} / \mathbf{N}$ & 0.18 & 0.16 & -0.24 & 0.38 & 0.04 & -0.45 & 0.29 & -0.08 & 0.01 & 0.11 & -0.32 & 0.11 & -0.23 & -0.47 & 0.13 & 0.25 & -0.13 & -0.22 & 0.44 & 0.20 & 1.00 & 0.08 & -0.12 & 0.14 & 0.01 & -0.03 \\
\hline ave & -0.08 & 0.22 & 0.32 & 0.15 & -0.19 & -0.18 & 0.19 & 0.48 & 0.24 & -0.64 & 0.22 & -0.58 & 0.38 & 0.23 & 0.00 & -0.03 & 0.20 & 0.08 & 0.69 & 0.63 & 0.08 & 1.00 & -0.98 & -0.48 & 0.32 & -0.21 \\
\hline ind & -0.07 & -0.34 & 0.33 & -0.28 & 0.05 & 0.25 & -0.21 & -0.51 & -0.36 & 0.50 & -0.17 & 0.43 & -0.39 & -0.13 & -0.14 & 0.03 & -0.19 & 0.00 & -0.70 & -0.66 & -0.12 & -0.98 & 1.00 & 0.32 & -0.36 & 0.17 \\
\hline & 0.50 & 0.28 & .01 & 0.38 & 0.58 & -0.07 & -0.02 & -0.25 & 0.25 & 0.77 & -0.12 & 0.81 & -0.04 & -0.33 & 0.44 & -0.07 & 0.00 & -0.21 & -0.30 & -0.31 & 0.14 & -0.48 & 0.32 & 1.00 & -0.36 & 0.33 \\
\hline clay & 0.41 & 0.49 & -0.06 & 0.32 & 0.19 & -0.49 & 0.10 & 0.50 & 0.52 & 0.00 & -0.31 & 0.02 & -0.02 & -0.43 & 0.35 & 0.10 & -0.22 & -0.33 & 0.47 & 0.55 & 0.01 & 0.32 & -0.36 & -0.36 & 1.00 & -0.22 \\
\hline Water & 0.22 & 0.22 & -0.09 & 0.40 & 0.59 & 0.17 & -0.31 & -0.44 & 0.13 & 0.31 & 0.06 & 0.47 & -0.13 & 0.21 & 0.42 & -0.20 & 0.21 & 0.18 & -0.38 & -0.42 & -0.03 & -0.21 & 0.17 & 0.33 & -0.22 & 1.00 \\
\hline
\end{tabular}

In bold significant correlations with $\mathrm{p}<0.05$. 


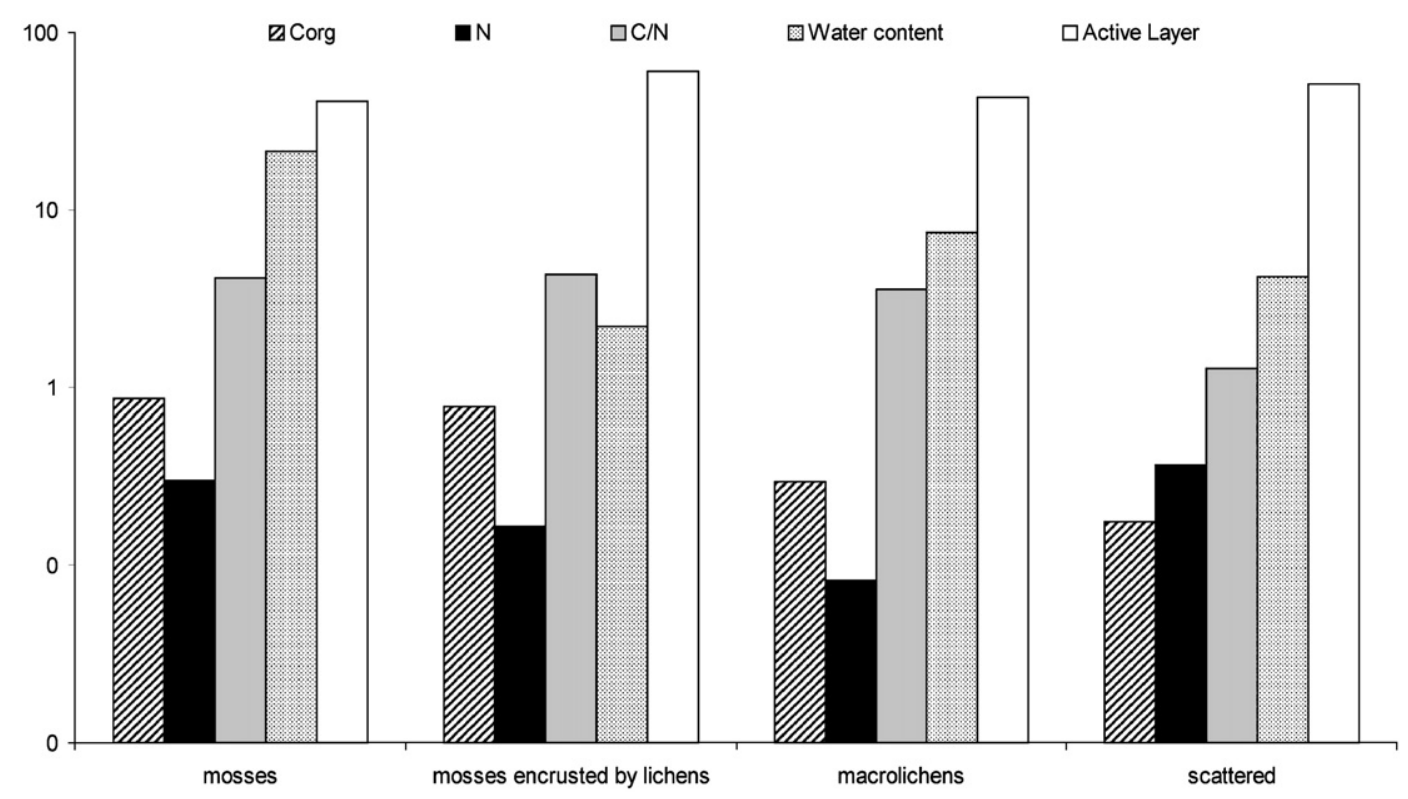

Fig. 6. Average values of Water content (\%); Active Layer depth (cm); Corg (\%); N (\%); C/N for different vegetation types (mosses, mosses encrusted by lichens, macrolichens, scattered crustose lichens).

been recognized to be key components closely interacting with the terrestrial ecosystems (e.g. Shiklomanov and Nelson, 2003; Walker et al., 2003; Chapin et al., 2005). Our results indicate that, even in continental Antarctica, vegetation type is able to influence both soil properties, particularly nitrate, organic carbon, $\mathrm{C} / \mathrm{N}$ ratio, water content, and active layer depth.

The higher concentrations of organic $\mathrm{C}$, nitrate and total $\mathrm{N}$ in the surface layers of the vegetated soils are expected results, as is the lower $\mathrm{pH}$, because of the accumulation of organic matter and its very low decomposition rates. The lower content of anions and cations in the upper layers of most vegetated soils could be related to the macronutrient uptake by cryptogams.

To assess the validity of our results in the Antarctic context, we compared our data with those from the available literature referring to the Victoria Land area, to the Dry Valleys and to other sites of Continental Antarctica. At Edmonson Point, Bargagli et al. (1999) analyzed soil characteristics along a transect from xeric bare ground to the centre of a wet moss stand in a site located very close to our study sites. In particular, the moss site (sample C) and the wet moss site (sample D) of Bargagli et al. (1999) are very similar to our permanent plots 1 (EP1) and 2 (EP2) in their location and vegetation characteristics. With respect to our results, the electric conductivity and, to a lesser extent, $\mathrm{pH}$ of those authors showed higher values in all conditions, whereas we recorded similar values of water content and $\mathrm{N}$, indicating that these parameters did not change in the period 1995-2003. Higher $\mathrm{pH}$ values, $\mathrm{C} / \mathrm{N}$ ratio, total organic carbon and similar $\mathrm{N}$ were obtained by Bargagli et al. (1996) on vegetated soils on the fumaroles of Mount Rittmann, a site located to the north of Edmonson Point and characterized by basaltic lavas.

Other available data from southern Victoria Land refer to the Dry Valleys, where the soils are generally dramatically more xeric than in our study sites (except for some samples collected at Boulder Clay, Prior Island, and Starr Nunatak) and are much more basic (except for some peculiar samples collected at Finger Point and Prior Island) (Barrett et al., 2006a). The $\mathrm{C} / \mathrm{N}$ ratio indicates much lower values in our study sites (ranging between 0.3 and 7.4) with respect to the Dry Valleys (ranging between 9.2 and 87.8) (Hopkins et al., 2006). The lower $\mathrm{C} / \mathrm{N}$ ratio and the higher water content of our soils show that, along the investigated transect, the decomposition processes of the organic matter seem to be more active than in the Dry Valleys and not so limited by soil moisture, although further analyses are still required to measure the decomposition rates at Victoria Land.

These results indicate that our data may be considered representative of the variability of the chemical and physical properties of Victoria Land soils, covering a wide range of sites, lithology and latitude and then providing a starting point for the large scale assessment of the soil characteristics of the long term monitoring network. In addition, the comparison with the data from the Dry Valleys confirms the appropriateness of considering Victoria Land separately from the Dry Valleys, and confirms that these two contiguous areas are driven by very different environmental forces/factors.

We also tested the significance of our sites within continental Antarctica, selecting the Wilkes Land area as a template, both because of the similar conditions of substrate (mainly acid rocks), latitude, vegetation, and of the availability of a critical mass of data comparable with ours. In particular, the study carried out by Melick and Seppelt (1997) focussing on the relations between soil properties and vegetation types allowed the comparison of $\mathrm{pH}$, conductivity, $\mathrm{N} \%$ and water content $\%$ of similar vegetation types with similar floristic composition, but located in geographically remote areas. At Wilkes Land, the soils are generally more acid and show higher moisture (averages ranging between $4.8 \%$ and $27.2 \%$ ) than at Victoria Land, independently of the vegetation type. The moisture patterns related to vegetation type are slightly different. The highest moisture is recorded under the moss communities, like 
at Victoria Land, but the macrolichen vegetation dominated by Usnea sphacelata shows the lowest values, unlike in Victoria Land where the lowest water content was measured under the mosses encrusted by lichens. Despite their more northern latitude, it is remarkable that the soils of Victoria Land accumulate higher contents of $\mathrm{N}$, especially in the moss community (averages 0.05 at Wilkes Land and 0.3 at Victoria Land) and also in the mosses encrusted by lichens (average range 0.030.06 at Wilkes Land and average 0.17 at Victoria Land).

Measurements carried out by Beyer et al. (2000) at Wilkes Land confirmed the more acid $\mathrm{pH}$ of the soils and indicated a higher $\mathrm{C} / \mathrm{N}$ ratio (ranging from 5.3 to 35.8 ) than at Victoria Land, despite the greater water availability, northern latitude and milder macroclimatic conditions of Wilkes Land.

\section{Conclusions}

The description of the main physical and chemical parameters of the soils within the monitoring network here presented may be a suitable reference for future monitoring activities in Victoria Land. Our results demonstrated that, at least for the analyzed samples, the latitudinal gradient does not significantly affect the chemical and physical properties of the soil. In fact, local microclimatic conditions seem to be more effective than the regional gradient, as was demonstrated for active layer depth (Guglielmin, 2006) and vegetation distribution (Cannone, 2006).

Lithology is able to influence some selected chemical parameters $(\mathrm{Al}, \mathrm{Fe}, \mathrm{Ca}, \mathrm{K})$ only to a small extent, while grain size and active layer depth do not show any recognizable impact. The occurrence of ornithogenic soils seems to be more effective than all the other factors in influencing the soil properties. On the other hand, the vegetation types are able to influence the properties of the surface layers of the soil (nitrate, organic carbon, $\mathrm{C} / \mathrm{N}$ ratio and water content) and are related to active layer depth.

Further analyses on soil properties including a greater number of sites, which would represent lithological variability more extensively, are still required, as is an extension of the latitudinal extremes of the gradient.

\section{Acknowledgements}

The authors wish to thank PNRA (Progetto Nazionale di Ricerca in Antartide), for providing the opportunity to undertake this research, and the logistical support within the PNRA project "Permafrost and Global Change in Antarctica" (PNRA project 5.3) led by Prof. Mauro Guglielmin. We also thank Antje Eulenburg and Heiko Baschek (both of the Alfred Wegener Institute for Polar and Marine Research) for their technical support with analyses of the samples. The English revision of the manuscript was carried out by Prof. James Gilbert Burge.

\section{References}

Bargagli, R., Broady, P.A., Walton, D.W.H., 1996. Preliminary investigation of the thermal biosystem of mount Rittmann fumaroles (northern Victoria Land, Antarctica). Antarctic Science 8 (2), 121-126.
Bargagli, R., Smith, R.I.L., Martella, L., Monaci, F., Sanchez-Hernandez, J.C., Ugolini, F.C., 1999. Solution geochemistry and behaviour of major and trace elements during summer in a moss community at Edmonson Point, Victoria Land, Antarctica. Antarctic Science 11 (1), 3-12.

Barrett, J.E., Virginia, R.A., Wall, D.H., Cary, S.C., Adams, B.J., Hacker, A.L., Aislabie, J.M., 2006. Co-variation in soil biodiversity and biogeochemistry in northern and southern Victoria Land, Antarctica. Antarctic Science 18 (4), 535-548.

Barrett, J.E., Virginia, R.A., Parsons, A.N., Wall, D.H., 2006. Soil carbon turnover in the McMurdo Dry Valleys, Antarctica. Soil Biology \& Biochemistry 38, 3065-3082.

Beyer, L., Bolter, M., Seppelt, R., 2000. Nutrient and thermal regime, microbial biomass and vegetation pattern of Antarctic cryosols in the coastal region of East Antarctica Wilkes Land. Arctic, Antarctic and Alpine Research 32, 30-39.

Bockheim, J.G., 2002. Landform and soil development in the McMurdo Dry Valleys, Antarctica: a regional synthesis. Arctic, Antarctic and Alpine Research 34 (3), 308-317.

Bockheim, J.G., 2008. Functional diversity of soils along environmental gradients in the Ross Sea region, Antarctica. Geoderma 144, 32-42.

Bunt, J.S., Rovina, A.D., 1955. Microbiological studies of some subantarctic soils. Journal of Soil Science 6, 119-128.

Cannone, N., 2004. Minimum area assessment and different sampling approaches for the study of vegetation communities in Antarctica. Antarctic Science 16, 157-164.

Cannone, N., 2005. Moss and Lichen flora of Victoria Land (Continental Antarctica) along a latitudinal transect. Terra Antarctica Report 11, 1-9.

Cannone, N., 2006. A network for monitoring terrestrial ecosystems along a latitudinal gradient in Continental Antarctica. Antarctic Science 18 (4), 549-560.

Cannone, N., Guglielmin, M., 2003. Vegetation and permafrost: sensitive systems for the development of a monitoring program of climate change along an Antarctic transect. In: Huiskes, A.H.L., Gieskes, W.W.C., Rozema, J., Schorno, R.M.L., Van der Vies, S.M., Wolff, W.J. (Eds.), Antarctic Biology in a Global Context. Backhuys Publishers, Leiden, pp. 31-36.

Cannone, N., Ellis Evans, J.C., Strachan, R., Guglielmin, M., 2006. Interactions between climate, vegetation and active layer in Maritime Antarctica. Antarctic Science 18 (3), 323-333.

Castello, M., Nimis, P.L., 1995. The lichen vegetation of Terra Nova Bay (Victoria Land, Continental Antarctica). Bibliotheca Lichenologica 58, 43-55.

Chapin III, F.S., Sturm, M., Serreze, M.C., et al., 2005. Role of land-surface changes in Arctic summer warming. Science 310, 657-660.

Convey, P., 2001. Terrestrial ecosystem response to climate changes in the Antarctic. In: Walther, G.-R., Burga, C.A., Edwards, P.J. (Eds.), Fingerprints' of climate change - adapted behaviour and shifting species ranges. Kluwer, New York, pp. 17-42.

Cook, A.J., Fox, A.J., Vaughan, D.G., Ferrigno, J.G., 2005. Retreating glacier fronts on the Antarctic Peninsula over the past half-century. Science 308, 541-544.

Day, P.R., 1965. Particle fractionation and particle-size distribution. In: Black, C.A., et al. (Ed.), Methods of soil analysis. Part I. Agronomy, vol. 9. American Society of Agronomy, Madison, Wisc., pp. 545-566.

Doran, P.T., Priscu, J.C., Barry Lyons, W., et al., 2002. Antarctic climate cooling and terrestrial ecosystem response. Nature 415, 517-520.

Duphorn, K., 1981. Physiographical and glaciogeological observations in north Victoria Land, Antarctica. Geolosche Jahrbuch B41, 89-109.

Gerasimov, I.P., Glazovskaya, M.A., 1965. Fundamentals of Soil Science and Soil Geography. Israel Program for Scientific Translation.

Geringhausen, U., Bräutigam, K., Osama, M., Hans-Ulrich, P., 2003. Expansion of vascular plants on an Antarctic island - a consequence of climate change? In: Huiskes, A.H.L., Gieskes, W.W.C., Rozema, J., Schorno, R.M.L., Van Der Vies, S.M., Wolff, W.J. (Eds.), Antarctic biology in a global context. Backhuys, Leiden, pp. 79-84.

Grigioni, P., De Silvestri, L., Pellegrini, A., Sarao, L., 1992. Some climatological aspects in the Terra Nova Bay Area, Antarctica. In: Colacino, M., Giovanelli, G., Stefanutti, L. (Eds.), Italian Research On Antarctic Atmosphere. Sif, Bologna, pp. 97-121. 
Guglielmin, M., 2006. Ground surface temperature (GST), active layer, and permafrost monitoring in continental Antarctica. Permafrost and Periglacial Processes 17 (2), 133-143.

Guglielmin, M., French, H.M., 2004. Ground ice in the Northern Foothills, Northern Victoria Land, Antarctica. Annals of Glaciology 39, 495-500.

Guglielmin, M., Ellis Evans, J.C., Cannone, N., 2005. Interactions between climate, vegetation and active layer in Maritime and Continental Antarctica for Climate Change monitoring. Terra Antarctica Report 11, 15-27.

Guglielmin, M., Ellis Evans, C.J., Cannone, N., 2008. Active layer thermal regime under different vegetation conditions in permafrost areas. A case study at Signy Island (Maritime Antarctica). Geoderma 144, 73-85.

Harper, A., unpublished. 2006/07 Immediate Science Report: K089 Climate Data Acquisition - Scott Base and Arrival Heights. Antarctica New Zealand (unpublished internal report).

Hopkins, D.W., Sparrow, A.D., Elberling, B., Gregorich, E.G., Novis, P.M., Greenfield, L.G., Tilston, E.I., 2006. Carbon, nitrogen and temperature controls on microbial activity in soils from an Antarctic Dry Valley. Soil Biology and Biochemistry 38, 3130-3140.

Kappen, L., 1985. Vegetation and ecology of ice-free areas of Northern Victoria Land, Antarctica. 1. The lichen vegetation of Birthday Ridge and an Inland Mountain. Polar Biology 4, 213-225.

Mack, M.C., Schuur, E.A.G., Bret-Harte, M.S., Shaver, G.R., Chapin III, F.S., 2004. Ecosystem carbon storage in arctic tundra reduced by long-term nutrient fertilization. Nature 431, 440-443.

Melick, D.R., Seppelt, R.D., 1997. Vegetation patterns in relation to climatic and endogenous changes in Wilkes Land, Continental Antarctica. Journal of Ecology 85, 43-56.

Nelson, F.E., Outcalt, S.I., Brown, J., Shiklomanov, N.I., Hinkel, K.M., 1998. Spatial temporal attributes of the active layer thickness record, Barrow, Alaska, USA. In: Lewkowicz, A.G., Allard, M. (Eds.), Permafrost, Seventh International Conference, June 23-27, 1998, Proceedings, 57. Universite' Laval, Quebec City, Nordicana, pp. 797-802.

Oechel, W.C., Vourlitis, G.L., Hastings, S.J., Zulueta, R.C., Hinzman, L., Kane, D., 2000. Acclimation of ecosystem $\mathrm{CO}_{2}$ exchange in the Alaskan Arctic in response to decadal climate warming. Nature 406, 978-981.

Schwarz, A.M.J., Green, T.G.A., Seppelt, R.D., 1992. Terrestrial vegetation at Canada Glacier, Southern Victoria Land, Antarctica. Polar Biology 12, $397-404$.
Seppelt, R.D., Green, T.G.A., 1998. A bryophyte flora for Southern Victoria Land, Antarctica. New Zealand Journal of Botany 36, 617-635.

Seppelt, R.D., Green, T.G.A., Schroeter, B., 1995. Lichens and mosses from the Kar Plateau, Southern Victoria Land, Antarctica. New Zealand Journal of Botany 33, 203-220.

Seppelt, R.D., Green, T.G.A., Schroeter, B., 1996. Additions and corrections to the lichen flora of the Kar Plateau, Southern Victoria Land, Antarctica. New Zealand Journal of Botany 34, 329-331.

Shiklomanov, N.I., Nelson, F.E., 2003. Statistical representation of landscape-specific active-layer variability. Proceedings of the Eighth International Conference on Permafrost, 21-25 July 2003. Balkema, Zurich, pp. 1039-1044.

Smith, R.I.L., 1999. Biological and environmental characteristics of three cosmopolitan mosses dominant in continental Antarctica. Journal of Vegetation Science 10, 231-242.

Soil Survey Staff, 2006. Keys to Soil Taxonomy,10th ed. US Department of Agricolture, Natural Resources Conservation Service, Washington D. C.

ter Braak, C.J.F., Šmilauer, P., 1998. CANOCO. Reference manual and user's guide to CANOCO for windows. Software for Canonical Community Ordination (version 4). Centre for Biometry, Wageningen.

Turner, J., Colwell, S.R., Marshall, G.J., Lachlan-Cope, T.A., Carleton, A.M., Jones, P.D., Lagun, V., Reid, P.A., Iagovkina, S., 2005. Antarctic climate change during the last 50 years. International Journal of Climatology 25, 279-294.

Vaughan, D.G., Marschall, G.J., Connolley, W.M., et al., 2003. Recent rapid regional climate warming on the Antarctic Peninsula. Climatic Change 60, 243-274.

Walker, D.A., Jia, G.J., Epstein, H.E., et al., 2003. Vegetation-soil-thaw-depth relationships along a low-Arctic bioclimate gradient, Alaska: Synthesis of information from the ATLAS studies. Permafrost and Periglacial Processes 14, 103-123.

Wynn-Williams, D.D., 1985. The biota of a lateral moraine and hinterland of the Blue Glacier, South Victoria Land, Antarctica. British Antarctic Survey Bulletin 66, 1-5. 